NIST

PUBLICATIONS

\section{New Concepts of \\ Precision Dimensional \\ Measurement for \\ Modern Manufacturing}

Dennis A. Swyt

U.S. DEPARTMENT OF COMMERCE National Institute of Standards and Technology

Manufacturing Engineering Laboratory Gaithersburg, MD 20899
U.S. DEPARTMENT OF COMMERCE Robert A. Mosbacher, Secretary NATIONAL INSTIUUTE OF STANDARDS AND TECHNOLOGY John W. Lyons, Director

$-\mathrm{QC}$

C. 2 

New Concepts of Precision Dimensional Measurement for Modern Manufacturing

\section{Dennis A. Swyt}

U.S. DEPARTMENT OF COMMERCE National Institute of Standards and Technology

Manufacturing Engineering Laboratory Gaithersburg, MD 20899

August 1991

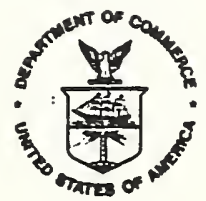

U.S. DEPARTMENT OF COMMERCE Robert A. Mosbacher, Secretary NATIONAL INSTIUUTE OF STANDARDS AND TECHNOLOGY

John W. Lyons, Director 



\section{TABLE OF CONTENTS}

page

I. Introduction 1

II. The Scope of Dimensional Measurements 2

A. The Range of Dimensional Measurements

B. The Types of Dimensional Measurements

III. The Basis of Dimensional Measurements

8

A. Realization of the Unit of Length

B. The Transfer of the Metric to Physical Objects

C. Standards-Imposed Limits to Accuracy

IV. Assessing Dimensional Measurements

A. Statistical Characterization of Error

B. Formal-Theoretical Characterization of Error

V. Principal Specific Sources of Errors

A. Error in Displacement Interferometry

B. Error in Coordinate-Position Measurement

C. Error in Distance Measurement

D. Error in Extension Measurement

E. Compounding of Error in Measurement Types

VI. Error-Budget Example

A. Displacement Errors

B. Error in Position Measurement $\Delta \mathrm{P}$

C. Error in Distance Measurement $\Delta \mathrm{d}$

D. Error in Extension Measurement $\Delta \mathrm{e}$

E. Summary and Analysis of Errors

VII. Conclusion 50

$\begin{array}{lr}\text { Acknowledgments } & 50\end{array}$

$\begin{array}{ll}\text { Appendix A } & 51\end{array}$

References $\quad 53$ 



\section{NEW CONCEPTS OF PRECISION DIMENSIONAL MEASUREMENT FOR MODERN MANUFACTURING}

\section{DENNIS A. SWYT}

Precision Engineering Division National Institute of Standards and Technology

Gaithersburg, Maryland 20899

\section{INTRODUCTION}

A hallmark of modern products is the high precision of the dimensions of their functional parts - high, that is, compared to that of their less-modern contemporaries. Since the Industrial Revolution, such has been the case. Modern products of the last century included certain factory-produced small arms, the precision of whose parts allowed them to be interchangeably assembled in the then-new system of mass production. Modern products of this century are video cassette recorders, the precision of whose parts allows read-write heads to be aerodynamically flown over the recording medium at microscopic altitudes.

Since it is modern products which sell best, for U.S. manufacturing firms seeking to compete better against evermore sophisticated foreign producers, the ability to realize precision higher than that of their competitors is essential. Precision, however, is relative not only to the capabilities of the competition, but also to the size of the functional parts in question. Comparable degrees of high precision are a common end for a wide range of manufacturers, from producers of the very large, such as commercial aircraft, to producers of the very small, such as nanoelectronic devices. 
This chapter presents a new scheme for the analysis of the dimensional capabilities of measuring machines and machine tools aimed at the realization of higher degrees of precision in the manufacture of dimensioned parts. Successive sections deal with the range and types of dimensional measurements, their relation to the basic unit of measure, the means for making such measurements, and the errors associated with them. Application of these principles is illustrated by a detailed analysis of the use of a laserbased coordinate measuring machine for the measurement of a physical part. Also included is an indication of how the identical analysis may be applied to a machine tool which shapes such parts.

\section{THE SCOPE OF DIMENSIONAL MEASUREMENTS}

Manufacture of today's most modern products requires the ability to carry out precise dimensional measurements over a wide scope, one which includes subtly different types and astonishingly different scales. Dimensional measurements of all types and scales, however, are expressed in terms of the basic unit of length.

\section{A. The Range of Dimensional Measurements}

The range over which precision dimensional measurements for the characterization of manufactured goods are made today spans nearly twelve orders of magnitude, segmented into relatively well recognized regimes:

\section{The Macro-Scale}

On the large-size end of the range are products which are not so much manufactured as constructed, that is, large-scale products often assembled on site rather than manufactured on line. Macro-scale products such as ships, aircraft, and spacecraft can have longest dimensions of the order of a hundred meters.

\section{The Mid-Scale}

In the mid-size of the range are products the parts of which are manufactured and assembled in the familiar factory environment, including a host of commonplace products from automobiles to machine screws. Mid-scale products have characteristic dimensions of the order of multiple- to fractionalmeters. 


\section{The Micro-Scale}

On the conventional small-size end of the range are products which are manufactured within special machines such as optical, electron-beam, and x-ray lithography systems. Less familiar products such as ultra-large-scale-integration (ULSI) microelectronic devices. Micro-scale products have characteristic dimensions often of less than a micrometer.

\section{The Nano-Scale}

Just emerging now is technology for the manufacture of products of the "nanoscale," including nanoelectronic (as opposed to microelectronic) transistor-type devices 100 times smaller than those in present commercial production and mechanical devices such as ultra-miniature pumps capable of implantation into blood vessels and automatically metering out drugs. Nano-scale structures and devices have features composed of small numbers of atoms and molecules and have characteristic dimensions of the order of nanometers $\left(10^{-9} \mathrm{~m}\right.$ or 40 billionths of an inch). Achievement of high precision in dimensional measurements at the nanometer scale demands utmost attention to physicalscientific, engineering and metrological principles [1].

\section{B. The Types of Dimensional Measurements}

In the characterization of today's most modern manufactured products, precision dimensional measurements are carried out not only over a wide range of dimensions but over a range of types, each of which, while intimately related to the others, has a uniquely distinct role in measurement.

\section{Position}

a. Geometrical Concept of Position: In geometry, position is the location of a point relative to either: another point, such as the origin of a one-dimensional coordinate system; a line, such as an axis of a two-dimensional coordinate system; or a plane, such as the reference surface in a three-dimensional coordinate system. For a three-dimensional cartesian system, the formally explicit expression for geometric position is a vector quantity:

$$
p=p(x x, y y, z z)
$$

where $x, y$, and $z$ represent the coordinates along unit vectors $x, y, z$ relative to the (suppressed null-vector) origin of the coordinate system. 
b. Physical Concept of Position: In physics, position is the location in space of a physical body. In the rigid-body approximation, where the configuration of that body is fully specified by six generalized coordinates (three linear and three rotational) corresponding to its six degrees of freedom, position is given by the three linear coordinates. Note that since both a rigid body and a reference frame have the same six degrees of freedom, locating a body relative to a reference frame is fully equivalent to locating one rigid body relative to another. In a three-dimensional cartesian system, the formally explicit expression for physical position is a vector quantity:

$$
P=P(X X, Y Y, Z Z)
$$

where $X, Y$, and $Z$ represent the coordinates of the location of one physical body relative to the location of another physical body which acts as the reference frame of the coordinate system.

c. Measurement Concept of Position: In measurements, position is the assigned numerical value of the length of path between the single point which describes the location of a physical body and the origin, axis, or plane of the one-, two-, or three-dimensional coordinate system expressed in terms of the standard unit of measure. In a three-dimensional cartesian system, the formally explicit expression for measurement position is a vector quantity:

$$
\mathbf{P}=\mathbf{P}(\mathrm{XX}, \mathrm{YY}, \mathrm{ZZ})
$$

where $\mathrm{X}, \mathrm{Y}$, and $\mathrm{Z}$, expressed in meters, represent the coordinates of the location of one physical body relative to the location of another physical body which acts as the reference frame of the coordinate system. Measurements of position are made, for example, in precision surveying of geodetic coordinates relative to control points and in measurements of the locations of points on objects by means of coordinate measuring machines.

\section{Displacement}

a. Geometrical Concept of Displacement: In geometry, there is nothing fully equivalent to displacement. However, the physical notion of displacement does involve the geometrical notion of translation, where translation is the transformation of a point at one position into a point at a different position by means of the operation:

$$
t=p_{\mathrm{f}}-p_{\mathrm{o}}
$$


where $t$ is the vector of translation and $p_{\mathrm{o}}$ and $p_{\mathrm{f}}$ are the initial and final position vectors of the point.

b. Physical Concept of Displacement: In physics, displacement is the change in location in space of a physical body with time, that is, its movement from one location to another, and is described by:

$$
T=P_{\mathrm{f}}\left(\mathrm{t}_{1}\right)-P_{\mathrm{o}}\left(\mathrm{t}_{2}\right)
$$

where $T$ is the translation representing the change in position with time of a single body from position $\boldsymbol{P}_{\mathrm{o}}\left(\mathrm{t}_{1}\right)$ at time one to position $\boldsymbol{P}_{\mathrm{f}}\left(\mathrm{t}_{2}\right)$ at time two.

c. Measurement Concept of Displacement: In measurements, displacement is the assigned numerical value of the length of path between the location of a physical body at an initial time and its location at a final time expressed in terms of the standard unit of measure. In a three-dimensional cartesian system, the formally explicit expression for measured displacement $\mathbf{D}$, expressed in meters, would be a vector quantity:

$$
\mathbf{D}=\mathbf{P}_{\mathrm{f}}\left(\mathrm{t}_{1}\right)-\mathbf{P}_{\mathrm{o}}\left(\mathrm{t}_{2}\right)
$$

where the difference between $P_{0}\left(t_{1}\right)$ and $P_{f}\left(t_{2}\right)$ represents the change in location, between times one and two, of one physical body relative to the location of another physical body (which acts as the reference frame of the coordinate system). Measurements of displacement are made, for example, in the characterization of the travel of a stage by means of a laser interferometer, where the stationary parts of the interferometer comprise the body which defines the reference frame.

\section{Distance}

a. Geometrical Concept of Distance: In geometry, distance is the separation between two points. In a three-dimensional cartesian system, the formally explicit expression for geometric distance would be a scalar quantity:

$$
d=\left|p_{1}\left(x_{1} x, y_{1} y, z_{1} z\right)-p_{2}\left(x_{2} x, y_{2} y, z_{2} z\right)\right|
$$

where $\mathrm{x}, \mathrm{y}$, and $\mathrm{z}$ represent the values of measured coordinates relative to the usually-suppressed null-vector origin of the coordinate system. 
b. Physical Concept of Distance: In physics, distance is the separation in space of the locations of two objects. In a three-dimensional cartesian system, the formally explicit expression for physical distance would be a scalar quantity:

$$
d=\left|P_{1}\left(X_{1} X, Y_{1} Y, Z_{1} Z\right)-P_{2}\left(X_{2} X, Y_{2} Y, Z_{2} Z\right)\right|,
$$

where $d$ is the distance between two bodies located at positions $P_{1}$ and $P_{2}$ respectively.

c. Measurement Concept of Distance: In measurements, distance is the assigned numerical value of the length of path between the location of one physical body and the location of another, expressed in the standard unit of measure. A formally explicit expression for measured distance, expressed in meters, would be a scalar quantity:

$$
d=\left|P_{1}\left(t_{1}\right)-P_{2}\left(t_{1}\right)\right|,
$$

where $\mathbf{P}_{1}\left(\mathrm{t}_{1}\right)$ and $\mathbf{P}_{2}\left(\mathrm{t}_{1}\right)$ represent the locations of two different bodies at the same time. Measurements of distance are made, for example, in the center-tocenter separation of engine cylinders and microelectronic circuit elements and in the calibration of the spacings of the successive graduations of various types of what are usually called "length scales," ranging from hundred-meter-long survey tapes to micrometer-long "pitch" standards for optical- and electronmicroscope measurement systems.

\section{Extension}

a. Geometrical Concept of Extension: In geometry, extension is the line segment between two points which lie on a surface which is topologically equivalent to a sphere such that all points on the line segment near the surface are either interior to or exterior to the surface. Equivalently, extension is the line segment connecting two points on a surface which have exterior normals with components that are anti-parallel. A formally explicit expression for physical extension $e$ is:

$$
e=\left|r_{\mathrm{b} 1}(\psi)-r_{\mathrm{b} 2}\left(\psi+180^{\circ}\right)\right|,
$$

where $r_{\mathrm{b} 1}(\psi)$ and $r_{\mathrm{b} 2}\left(\psi+180^{\circ}\right)$ represent the locations of points on a surface having exterior normals $180^{\circ}$ apart. 
b. Physical Concept of Extension: In physics, extension is the amount of physical space that is occupied by, or enclosed by, a material object. A formally explicit expression for physical extension is:

$$
E=\left|R_{\mathrm{b} 1}(\psi)-R_{\mathrm{b} 2}\left(\psi+180^{\circ}\right)\right|,
$$

where $R_{\mathrm{b} 1}(\psi)$ and $R_{\mathrm{b} 2}\left(\psi+180^{\circ}\right)$ represent respectively the locations of the opposite-facing boundaries of the physical object which, for convenience, may be expressed in terms of a vector radius from the centroid of the body.

c. Measurement Concept of Extension: In measurements, extension is the assigned numerical value of the length of path between the location of one boundary of a material object relative to the location of another oppositefacing boundary, expressed in terms of the standard unit of measure. A formal expression for measured extension is:

$$
\mathrm{e}=\left|\mathbf{P}_{\mathrm{b} 1}(\Psi)-\mathbf{P}_{\mathrm{b} 2}\left(\Psi+180^{\circ}\right)\right|
$$

where $\mathrm{e}$ is the extension and $\mathbf{P}_{\mathrm{b} 1}$ and $\mathrm{P}_{\mathrm{b} 2}$ represent the locations of oppositefacing boundaries of an object. Extension-type dimensions of objects include height, width, diameter, and also "length". Measurements of extension are made in general to achieve physical fit between mating parts. Prototypical examples of extension measurements are those in the automotive industry wherein the outside diameter of piston rings and the inside diameter cylinder walls are measured to insure slip fit. For analogous reasons, extension measurements are also made in the microelectronics industry on critical elements of microcircuit devices. Table 1 below summarizes key aspects of the various types of dimensional measurements just described.

In familiar terms, each type of "length" relates to a different, but interrelated, concern. For example, the "length" of an automobile (i.e. its extension) describe how big a parking space is needed, the "length" of a racetrack (i.e. its distance) describes how far it is between start and finish lines, and the "length" of a stage's travel (i.e. its displacement) describes how far its carriage can move. 
Table 1. Description of the Quantity and Nature of the Length of Path To Be Measured for Each of the Four Dimensional Measurement Types

\begin{tabular}{lll}
\hline $\begin{array}{l}\text { Dimension } \\
\text { Position }\end{array}$ & Quantity & The Length of Path Is That Between: \\
Visplacement $\mathbf{P}$ & $\begin{array}{l}\text { The location in space of a single object } \\
\text { and an origin of coordinates (equiva- } \\
\text { lent to a second, reference object) }\end{array}$ \\
Distance & Scalar d & $\begin{array}{l}\text { The locations of two objects } \\
\text { from time } \mathrm{t}_{1} \text { to time } \mathrm{t}_{2}\end{array}$ \\
Extension & Scalar e & $\begin{array}{l}\text { The locations of opposite-facing boun- } \\
\text { daries of an object }\end{array}$ \\
\hline \hline
\end{tabular}

\section{THE BASIS OF DIMENSIONAL MEASUREMENTS}

What "lengths" of all scales and types have in common is that each is measured and expressed in terms of a unit of length. For that unit to be the internationally standard unit of length, a formal definition must be operationally realized and transferred.

\section{A. Realization of the Unit of Length}

The function of a unit of length is to provide a metric for the physical space in which dimensional measurements are to be made. The function of a standard unit of length is to provide a metric which can be used in common in international science and trade.

\section{The Formal Definition of the Meter}

By treaty, the standard of length used in international science and trade is the meter. The meter is part of the International System of Units (SI) and is referred to as the fundamental, or SI, unit of length. Since 1983, the formal SI definition of the meter as the unit of length has been: 
"The meter is the length of path travelled by light in vacuum in the time interval 1 / 299792458 of a second" [2].

No longer based on lines scribed on a platinum-iridium "meter" bar (as it was from 1887 until 1960), the meter today is the distance which light travels in free space in (approximately) three billionths of a second.

\section{The Derived Nature of the Meter}

The new definition of the meter makes it in effect a derived quantity governed by the length-time relation:

$$
\mathrm{L}=\mathrm{c} \cdot \mathrm{T} \text {, }
$$

where $\mathrm{L}$ is the defined unit of length, $\mathrm{c}$ is the defined constant speed of light, and $T$ is the defined unit of time. The second is the SI unit of time and is defined in terms of a fixed number of counts of the frequency of oscillation of specific atomic states of a cesium-beam atomic clock.

\section{The Operational Realization of the Meter}

The meter is operationally realized through a chain of intercomparisons of the frequencies of various microwave-, infrared- and visible-wavelength lasers, based on the relationship of the frequency of electromagnetic waves and their wavelength:

$$
\mathrm{f} \cdot \lambda_{0}=\mathrm{c} \text {, }
$$

where $\mathrm{f}$ is frequency, $\lambda_{0}$ is vacuum wavelength, and $\mathrm{c}$ is value of the speed of light (that is, $2.99792458 \cdot 10^{8} \mathrm{~m} / \mathrm{sec}$ ). Based on this relationship, the ultimate limit on the uncertainty associated with the realization of the meter is that of the cesium clock, which is $10^{-13}$. At present, for dimensional measurements access in principle to the frequency cesium-beam atomic clock is through a chain of intercomparisons of wavelengths of microwave, infrared and visible sources by which the frequency of visible-wavelength lasers is determined.

\section{B. The Transfer of the Metric to Physical Objects}

In practical dimensional measurements, the means by which the SI unit of length as the metric of physical space is transferred to measurements of physical objects -- from automobile piston rings to microelectronic circuit elements - is through a system which successively involves visible light of known wavelength, displacement interferometry using that light, coordinate 
measurement systems based on that interferometry, and measurements on physical objects using such coordinate measurement systems.

\section{Visible Light of Known Wavelength}

For dimensional measurements, the practical primary standard of length today is the vacuum wavelength of the $633-\mathrm{nm}$ red-orange line of an iodineabsorption-stabilized helium-neon laser. One may realize the SI-unit-based wavelength, to a particular accuracy, by buying a commercial helium-neon laser stabilized to a corresponding degree.

\section{Displacement Interferometry}

The means by which propagating laser light, which defines the metric of physical space, is coupled to dimensional measurements in that space is through interferometric measurements of displacement.

The prototypical displacement interferometer is the two-beam, Michelson interferometer. The Michelson interferometer consists of a laser light source, beam splitter, fixed reflector (either a plane mirror or retroreflector), moving reflector and detector. In this type of interferometer the translation of the moveable reflector, which must be parallel to the axis of propagation of the laser light, is measured in terms of whole and fractional numbers of fringes generated by the interference of the beams in the stationary and moving arms of the interferometer.

The typical displacement interferometer of today employs the heterodyne principal for measuring the change in optical phase between the two beams by having one frequency-shifted with respect to the other. A widely-used commercial heterodyne interferometer employs a two-frequency laser and the displacement of a plane mirror or retroreflector is measured by means of two channels of detectors, doublers, counters, subtractors and a calculator [3].

By whatever means the phase difference between the reference and movingmirror beams is measured, the operational link between the SI-unit-carrying wavelength of visible light and a displacement measurement is through the interferometer equation:

$$
\mathrm{D}=\mathrm{L}_{1}-\mathrm{L}_{2}=\mathrm{N} \lambda_{0} / 2 \mathrm{n}_{\mathrm{m}} \cdot \cos \theta
$$

where $\mathrm{D}$ is the displacement, $\mathrm{L}_{1}-\mathrm{L}_{2}$ is the change in length (i.e., extension) of the interferometer cavity, $\lambda_{0}$ is the vacuum wavelength of the laser light used, $\mathrm{N}$ is the real number describing the counted integer-order and measured fringe-fraction of the interference, $\mathrm{n}_{\mathrm{m}}$ is the index of refraction of the medium, and $\theta$ is the angle between the optical axis of the interferometer and the 
direction of propagation of the incident light. In multi-pass fringe-fractioning interferometers:

$$
\mathrm{D}=\mathrm{M} \lambda_{0} / 2 \mathrm{nmK} \cdot \cos \theta,
$$

where $M$ is the counted integer-order and measured fringe-fraction of the interference, $m$ is the number of passes of the light through the interferometer, and $\mathrm{K}$ is the number of electronic sub-divisions of the interference fringes (typically a power of two ranging from 16 to 1024 depending on the system).

For the high-resolution commercial heterodyne interferometer system described above, the limit of resolution, that is, the least count in displacement measurement is about $\lambda / 400$ or $1.5 \mathrm{~nm}$. Under development now at NIST is a specially optimized heterodyne interferometer intended to realize a least count of twenty times higher, that is, $\lambda / 8000$ or $0.07 \mathrm{~nm}$.

Note that, as described here, in a laser displacement-interferometry system, it is the value of the vacuum wavelength of the laser light which provides the metric of physical space as the fundamental unit of length. It is the overall interferometer system doing the subdivision of fringes which provides the "scale," that is, the metric-less subdivisions of that unit. In common usage, however, displacement-measuring devices, such as interferometer systems, are often called "scales" and perform both of the functions described here: subdividing the axes (that is, providing the scale for an axis) and carrying the metric (that is, providing the standard unit of length, relative to which the "scales" have somehow been appropriately calibrated).

\section{The Coordinate Measurement System}

After the wavelength of laser light and displacement interferometry, the next link to dimensional measurements of physical objects is a coordinate measurement system. A coordinate measurement system is necessary for the measurement of "position," that is, the location of a single point in space.

To establish a coordinate system, one must establish the means for realizing the geometry for physical space, including: axes (one, two, or three depending on the dimensionality of the system desired; an origin (the null-vector point from which radiate the axes of the coordinate system); a scale along each of the axes (that is, the graduation of each axis into subdivisions); and a metric (that is, a single measure of distance in the space of the reference frame to which the graduations of the axes relate).

In modern, automation-oriented manufacturing, the means by which the geometry of the coordinate measurement system is realized and precision 
dimensional measurements are made, is the coordinate measuring machine (CMM). A coordinate measuring machine embodies a coordinate system by means of its essential functional components, which are its frame, scales, carriages and probes as outlined in Table 2.

Today's high-performance three-dimensional coordinate measuring machines take on a wide variety of forms depending on the specific way in which each of these functional components is embodied. Frames can be of the bridge or cantilever-arm type. Scales can be ruled-glass optical encoders or enclosed-path laser interferometers. Carriages can be translating and rotating tables and moving bridges. Probes can be touch-fire or analog mechanical-contact types or capacitive, optical, or machine-vision non-mechanical-contact types.

Table 2. The Principal Functional Components of a Coordinate Measuring Machine (as well as of a Machine Tool)

Frame

The frame is the geometry-generating structure, which is the means for embodying the origin, axes, and angular relationships which comprise a coordinate system.

Scales The scales are the displacement-measuring devices, which are the means for realizing the metric-based graduations along each axis of the frame.

Probe The probe is the sensor system, which is the means for linking the boundary of the object to be measured to the frame and scales; in a machine tool, the "probe" is a material-moving element rather than a material-locating element as it is in a measuring machine.

Carriage The carriage is the complete motion-generating system, which is the means for translating the object to be measured relative to the probe, frame and scales.

Whatever particular form a coordinate measuring machine takes on, its fundamental function is to provide measurements of locations in space, that is, of position. However, just as a manufacturer wants to buy holes not drills, users of CMMs in manufacturing want measurements of dimensions of objects, such as those of their products, not coordinates of locations in space. 


\section{The Physical Object To Be Measured}

The terminus of the series of linkages, which allow dimensional measurements on physical objects in terms of the SI unit of length, is the object itself. Now there are two different kinds of features on objects corresponding respectively to the distance-type and the extension-type of dimensional measurements described above.

a. Distance-Type Features of Objects: Distance-type features, include, for example, the center-to-center spacings of cylinders of an automobile or spacing of left-edges of successive microcircuit elements. While, in fact, always to some degree extended, distance-type features are treated as extensionless points. Thus, the distance between automobile cylinders is measured in terms of their centroids. Similarly, the distance between the moon and the earth is center to center. Measurements of distance type features are inherently, in effect, point to point.

b. Extension-Type Features of Objects: Extension-type features include, for example, the diameters of the automobile cylinders or the widths of the microcircuit elements. This type of feature must be treated as made up of boundaries that must be approached from opposite directions as in calipering, in which one locates two points, one on each and measures the "distance" between them. However, it is in the essential nature of extension measurements that these boundaries are opposite facing. As will be seen below, it is the practical and theoretical difficulty in locating properly such boundaries that makes extension the most difficult type of dimensional measurement.

In sum, successful measurement of, for example, the diameter of an automobile cylinder or the width of a microelectronic element in terms of the SI unit of length requires dimensional measurements of each type in succession: displacement, position, distance, and, finally, extension. The measure of success in this succession of measurements at each level and in total is accuracy achieved.

\section{Standards-Imposed Limits to Accuracy}

The accuracy in practical dimensional measurements depends on the availability of standards for the calibration of the measuring machines by which measurements are made. Attainable accuracy is ultimately limited by national standards laboratories in measurements involving a realization of the meter, 
displacement interferometry, reference coordinate measuring machines, and calibrated artifacts.

\section{The Limit of Realization of the Meter}

The ultimate limit to accuracy in practical dimensional measurements is the accuracy with which national standards laboratories can realize the definition of the meter. At present that limit is the uncertainty with which the visible wavelength of the iodine-stabilized HeNe laser is known, that is, $10^{-9}$ compared to the $10^{-13}$ of the atomic clock. The meter as the unit-of-length metric of that space can be accessed directly then through the vacuum wavelength of the $\mathrm{HeNe}$ laser. Table 3 below shows the effective wavelength and uncertainty associated with $\mathrm{HeNe}$ lasers stabilized by various means.

Table 3. Comparison of the Value and Associated Fractional Uncertainty of the Vacuum Wavelength of Helium-Neon Lasers of Various Degrees of Stabilization

\begin{tabular}{|c|c|c|}
\hline Wavelength Value & Uncertainty & $\underset{\text { Stabilization }}{\mathbf{T}} \underset{\mathbf{p}}{\mathbf{p}} \mathbf{e}$ \\
\hline 632. $991 \mathrm{~nm}$ & $10^{-6}$ & Free-running \\
\hline 632. 9914 & $10^{-7}$ & $\begin{array}{l}\text { Any one of a } \\
\text { number of }\end{array}$ \\
\hline 632. 99139 & $10^{-8}$ & $\begin{array}{l}\text { opto-electronic } \\
\text { techniques }\end{array}$ \\
\hline 632. 991398 & $10^{-9}$ & $\begin{array}{l}\text { Iodine Absorption } \\
\text { Cell }\end{array}$ \\
\hline
\end{tabular}

As indicated in Table 3, the fractional uncertainties $(\Delta \lambda / \lambda)$ associated with commercially available $\mathrm{HeNe}$ lasers range from the $10^{-6}$ of a free-running device, through the $10^{-7}$ specified stability of certain commercial lasers, and $10^{-8}$ short-term stability of some such devices, up to the $10^{-9}$ accuracy of the visible wavelength of the iodine-stabilized laser as defined in the documents associated with the definition of the meter and realized in laboratory devices. 


\section{The Limit of Displacement Interferometry}

After the ultimate limit to accuracy of dimensional measurements imposed by the realization of the meter in terms of the wavelength of visible light, the next limit to that accuracy is the degree to which measurements of displacement can be made by means of optical-wavelength interferometry.

Based on work by national standards laboratories, the limit to the accuracy of interferometric displacement measurements at a displacement of a meter is estimated to be: $10^{-4}$ in air uncompensated for pressure, temperature and humidity; $10^{-7}$ in fully compensated ambient air; and $10^{-8}$ in standard dry air. Based on results by a variety of workers, a reasonable estimate for the practical limit for displacement interferometry in vacuum is $10^{-10}$.

\section{The Limit of Coordinate Measurement}

The next factor to limit the accuracy of dimensional measurements after the realization of the meter and displacement interferometry is the ability to embody a coordinate system in a measuring machine. At present, the highestaccuracy coordinate measurement is achieved by national standards laboratories on special-purpose one- and two-axis measuring machines.

At the U. S. National Institute of Standards and Technology, a machine for the calibration of line scales over the range $1 \mu \mathrm{m}$ to $1 \mathrm{~m}$ has associated with it a total uncertainty of measurement $\mathrm{U}_{\mathrm{T}}$ given by:

$$
\mathrm{U}_{\mathrm{T}}=10 \mathrm{~nm}+10^{-7} \cdot \mathrm{s}_{\mathbf{z}} \text {, }
$$

where $s_{\mathbf{z}}$ is the separation of any two graduations on the scale. At one meter, the positional error of this machine is approximately $1 \cdot 10^{-7}$ [4]. For a twoaxis machine used for the calibration of grid plates up to $600 \mathrm{~mm}$ square, the corresponding positional error in routine use is $0.5 \mu \mathrm{m}$, corresponding to about $5 \cdot 10^{-7}[5]$.

Currently unmet is the need in the microelectronic industry for an accuracy of $50 \mathrm{~nm}$ accuracy over $250 \mathrm{~mm}$, that is, $2 \cdot 10^{\cdot 7}[6]$. Because of a trend to even more stringent requirements, for the regime of nanotechnology, there is under development at the NIST a "Molecular Measuring Machine"[7], which is a planar 50 -by-50 $\mathrm{mm}$ xy-coordinate measuring machine (with a $100 \mu \mathrm{m} \mathrm{z}$ axis) which has a design-goal positional capability of:

$$
\mathrm{U}_{\mathrm{T}}=1 \mathrm{~nm}=10^{-8} @ 70 \mathrm{~mm} .
$$




\section{The Limit of Physical Artifact Standards}

The limiting error in many practical dimensional measurements is the accuracy of artifact standards (that is, physical objects), dimensions of which have been calibrated by a national standards laboratory or by a secondary laboratory referenced to it. Accuracies representative of various types of artifact standards calibrated by NIST are shown in the Table 4 below.

Table 4. The Type, Range, and Accuracies of Some Dimensional Standards Calibrated by the NIST

\begin{tabular}{llll} 
Dimensional Std & Type & \multicolumn{1}{c}{ Range } & Accuracy \\
& & & \\
Survey Tapes & d & $1 \mathrm{~mm}$ to $300 \mathrm{~m}$ & $5.0 \mu \mathrm{m}+10^{-6} \cdot \mathrm{d}$ \\
Gauge Blocks & d & $1 \mathrm{~mm}$ to $0.5 \mathrm{~m}$ & $0.025 \mu \mathrm{m}$ to $0.15 \mu \mathrm{m}$ \\
Line Scales & d & $1 \mu \mathrm{m}$ to $1 \mathrm{~m}$ & $0.01 \mu \mathrm{m}+10^{-7} \cdot \mathrm{d}$ \\
Photomask Lines & e & $0.5 \mu \mathrm{m}$ to $20 \mu \mathrm{m}$ & $0.05 \mu \mathrm{m}$ \\
Polymer Spheres & e & $0.3 \mu \mathrm{m}$ to $3 \mu \mathrm{m}$ & $0.01 \mu \mathrm{m}$ \\
Thin-Film Steps & d & $0.02 \mu \mathrm{m}$ to $10 \mu \mathrm{m}$ & $0.003 \mu \mathrm{m}$ to $0.2 \mu \mathrm{m}$
\end{tabular}

Note that because of their idiosyncratic nature and the intractability of dealing with the very object-specific boundary-location errors, NIST calibrates extension-type objects, such as integrated-circuit photomask linewidths [8], only when the national interest justifies the major long-term theoretical and experimental effort required.

Note also that while NIST provides measurement services for calibrations of distance and extension (which involve associating the unit of length with locations on physical objects), it does not calibrate displacement or position (which involve associating the unit of length with a device-based process [9].

\section{ASSESSING DIMENSIONAL MEASUREMENTS}

As suggested earlier, manufacture of modern products demands dimensional measurements of not only exceptional precision, that is, the closeness together, but also of exceptional accuracy, that is, closeness to a true value [10].

Especially in high-technology products including, for example, microwave resonators for communication systems and $\mathrm{x}$-ray optics for microelectronic 
lithography, accuracy is required because the dimension of device features is dictated not just by design convention but by the laws of physics. As a result, dimensions of features must not only be very close to $a$ value but to the value.

This section looks at the assessment of dimensional measurements in terms of statistical characterization of error, formal-theoretical characterization of error, compounding of error and standards-imposed limits of error.

\section{A. Statistical Characterization of Error}

Physical measurement is itself a process, like manufacturing, which produces output. The output of a measurement process is numbers, that is, the measurement results. The quality of these numbers can be assessed and characterized by means of measurement statistics in terms of precision and accuracy. The manufacture of modern products demands dimensional measurements that are of both high precision and high accuracy, however one chooses to describe that combined state.

\section{Precision-vs-Accuracy}

a. Precision: As a concept, precision conveys the notion of closeness together. As such, it is the principal figure of merit for the achievement of higher quality in the sense of lower variability. Precision is conventionally represented numerically in terms of the standard deviation from the mean for a number of measurements presumed to be randomly drawn from a large population describable by a Gaussian distribution function. The standard deviation for a single such measurement $\sigma_{i}$ is given by:

$$
\sigma_{\mathrm{i}}=\left[\Sigma_{\mathrm{i}}\left(\ell_{\mathrm{i}}-\ell_{\mathrm{m}}\right)^{2} /(\mathrm{n}-1)\right]^{1 / 2},
$$

where $\ell_{\mathrm{m}}=\Sigma_{\mathrm{i}} \ell_{\mathrm{j}} / \mathrm{n}$ and the summations $\boldsymbol{\Sigma}_{\mathrm{i}}$ are over the number of measurements $n$. Where $\sigma_{i}$ is a measure of the precision of one measurement, $\sigma_{m}$ is a measure of the precision of the mean of $n$ measurements and is given by:

$$
\sigma_{m}=\sigma_{i} / n^{1 / 2}
$$

Precision is given in terms of either one-, two- or three-sigma values for single measurements, corresponding respectively to confidences of $68.3 \%, 95.5 \%$, and 99.7\%. National standards laboratories typically use $3 \sigma_{\mathrm{m}}$ for characterizing dimensional standards which they calibrate. Instrument manufacturers often use $2 \sigma_{m}$ for characterizing dimensional-measurement devices which they sell. 
b. Accuracy: As a concept, accuracy conveys the notion of closeness to a true value. As such, accuracy is the principal figure of merit for the achievement of interchangeability of components and physical operation in high-technology devices. In those situations, actual not relative dimensions determine functional performance. Accuracy is conventionally represented numerically in terms of an estimate of the maximum that a measurement would likely be different from a true or standard value. In metrological terms, accuracy is the estimate of the degree to which measurements are free from systematic error and is represented by the term s.e.

\section{Error or Total Uncertainty}

The metrological term for a combined measure of precision and accuracy is uncertainty or error. Total uncertainty includes a fully-described estimate of systematic error and fully-described measure of precision. NIST, along with a number of the national standards laboratories of other countries, typically uses the three-sigma of the mean in the assignment of a total uncertainty given by:

$$
\mathrm{U}_{\mathrm{T}}=\text { s.e. }+3 \sigma_{\mathrm{m}} \text {. }
$$

Obviously, informed judgment must be made in how to sum and combine systematic and random error contributions. For example, the magnitudes of systematic errors may be added algebraically, that is, as signed quantities, if the signs are definitely known or arithmetically, that is, without signs, if not. Random errors may be added in quadrature if known to be uncorrelated or arithmetically if not.

Throughout this chapter, the term "error" is used synonymously with uncertainty $U_{T}$ as described above and is denoted by the Greek character delta, upper case $(\Delta)$ for total-type and lower ( 8 ) for type-specific.

\section{Additive-Multiplicative Representation}

Dimensional-measurement errors from different sources can be either additive in nature, that is, appearing as incremental off-set, or they may be multiplicative, that is, appearing as a length proportional. Total error can be of the form:

$$
\Delta \ell=A+B \cdot \ell,
$$

where $\mathrm{A}$ is the additive error and $\mathrm{B}$ the multiplicative. 
a. Added and Multiplicative Components: Given a system in which errors from a number of sources are to be combined to estimate the total error in a lengthbased dimensional measurement of a given type, then:

$$
\Delta \ell=\left(\Sigma \delta \ell_{\mathrm{Ai}}\right)+\left(\Sigma \delta \ell_{\mathrm{Bi}}\right) \cdot \ell,
$$

where A now is the sum $\Sigma_{\mathrm{i}}$ over individual additive contributions $\delta \ell_{\mathrm{A}}$ and $\mathrm{B}$ the sum $\Sigma_{\mathrm{i}}$ over individual multiplicative contributions $\delta \ell_{\mathrm{Bi}}$ where $A_{\mathrm{i}}$ and $\mathrm{B}_{\mathrm{i}}$ can be comprised of either or both systematic errors and random errors.

b. Manufacturers' Statements of Accuracy: Many commercial producers of measuring machines conventionally quote an accuracy for the performance of their products in additive-multiplicative form, as in, for example:

$$
\Delta \mathrm{L}=2 \mu \mathrm{m}+[\mathrm{L}(\mathrm{mm}) / 500 \mathrm{~mm}] \mu \mathrm{m},
$$

which means that over the $1 \mathrm{~m}$-travel of the machine, the accuracy of single measurements of position would range from a minimum of $2 \mu \mathrm{m}$ to a maximum of $4 \mu \mathrm{m}$.

\section{B. Formal-Theoretical Characterization of Error}

Simple assessments and statements of accuracy which put errors into the simple additive-multiplicative form lump two types of additive errors, constant off-sets and non-linearities, and provide an inadequate basis for identification of the sources of error. For diagnostic purposes, the better approach is to consider the most general case, that of a dimensional measuring system that produces output which includes linear and non-linear terms in the form:

$$
l_{o b s}=a+(1+b) \cdot l_{t}+c\left(l_{t}^{2}\right)
$$

where $l_{b b}$ is the measured value, $l_{t}$ is the true length, $a$ and $b$ are constants and $c\left(\ell_{t}^{a}\right)$ is the sum of all non-linear terms. The error in measured values $\Delta \ell$ is then given by:

$$
\Delta \ell=l_{b b s}-\ell_{t}=a+b \cdot l_{t}+c\left(l_{t}^{p}\right) .
$$

Each of the error components $\mathrm{a}, \mathrm{b}$ and $\mathrm{c}$ describes a specific type of error source and is a measure of the degree to which the measurement system has realized a fundamental requirement of measurement. 


\section{The Fundamental Axioms of Measurement}

For dimensional measurements to yield physically meaningful results, they must conform to axiomatically fundamental requirements given in the Philosophical Foundations of Physics by Rudolf Carnap [11] and provided a more accessible account by Simpson [12]. These axiomatic requirements are that a measuring system must be able to rank order objects along the dimension of measurement and to reproduce properly the unit, the zero, and the scale of that dimension.

a. The Rank-Order Operator: The rank-order operator is the overall procedure by which objects or processes being measured are ordered and assigned values of the quantity being measured. In length-based dimensional measurements, the ordering operator is equivalent to a comparison of two objects involving, in effect, a translation in physical space. Each type of dimensional measurement, however, requires its own particular realization of that ordering operator. As a result, displacement, position, distance, and extension each constitutes its own measurement dimension which must be operationally tied to the dimension of physical space.

b. The Unit: The unit is the rank-order greater of two objects or states of the physical system assigned a defining numerical value of the quantity being measured. The unit is one of the two points required to specify what is axiomatically a linear system. In length-based dimensional measurements which conform to international standards, the unit is the SI unit of length. In practical dimensional measurements today, the unit of length is an internationallyaccepted value for a well-defined wavelength of an iodine-stabilized HeNe laser as discussed above in Section IIIA.

c. The Zero: The zero is the rank-order lesser of the two objects or states of the physical system assigned a defining numerical value of the quantity being measured. In effect, the zero is the other of the two points required to specify the linear system. Implied by the definition of the unit of length is that the zero of measurement has an assigned numerical value of zero (that is, $\left|\mathrm{l}_{z}\right|$ $\equiv 0$ ) and that the zero of length of dimensional measurements is the length of the path in space which light traverses in the zero interval of time.

In practical dimensional measurements, the zero corresponds to the physical zero-vector origin of coordinates which must be practically realized in each of the different dimensional types. For example, in laser interferometry, the zero of displacement is the initial optical-path difference between the reference beam and moving-mirror beam, one which must remain constant during subsequent translation of the mirror in order to prevent loss of origin of 
displacement. In coordinate measuring machines, the zero of position is the initial location of the tip of the probe relative to the interferometer reflector, which must remain constant as the probe is moved about to prevent loss of origin of position.

d. The Scale: The scale is the set of equal graduations into which the difference between the unit and the zero is divided. In effect, the scale is the condition of linearity under which differences in dimensions are equal, that is, the difference between one pair of objects is the same as the difference between another pair. Operationally, the scale is the means for measuring in increments smaller than the unit.

In practical dimensional measurements, the scale is the combination of a physical transducer and a linearization algorithm by which linear interpolations between known points can be carried out. For example, in displacement interferometry, the scale is generated by interpolating between fringes using an assumed sinusoidal function of waveforms for the linearization algorithm. Nonlinear transducers such as capacitance gauges -- when used in conjunction with suitable mathematical models of their responses -- can also be used to generate the required linear scale.

In sum, the axioms of measurement define the requisite elements of a measurement system, each of which must be operationally realized to achieve meaningful results. The first axiom - the rank-order operation - defines the dimension of measurement, such as temperature, time, voltage, or, in this case, each of the four types of length. The other three axioms are the means by which numerical values are associated with measurements of that dimension. Table 5 below shows explicitly the form of the axiom errors for each of the dimensional types.

\section{Analysis of Error in Terms of the Axioms}

Associated with each of the latter three axioms of measurement is a specific type of error with specific, fixable causes. Errors of the unit, the zero and the scale correspond respectively to the terms in Eq. 26 with $a$ equal to the error of the zero, $b$ to the error of the unit, and $c\left(l_{t}^{2}\right)$ to the error of the scale, that is, the sum of all non-linear terms.

a. Error of the Zero: Errors of the zero occur for measurement systems which have a properly linear relation of measured value to true value, $\ell_{o b s}$ to $\ell_{v}$, but have a non-zero value at $\ell_{t}=0$, that is: 
Table 5. The Matrix of Errors by Measurement Axiom and Dimensional Type in General Form

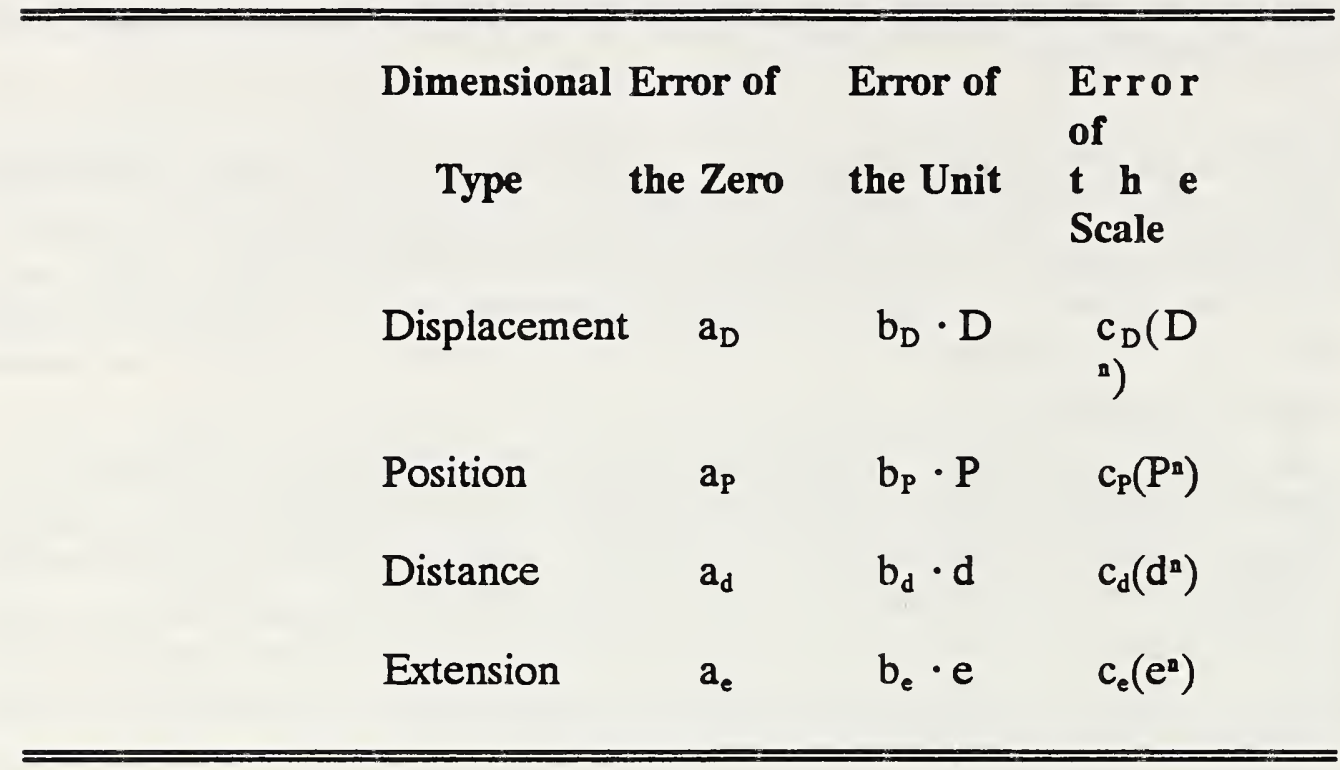

$$
l_{\text {obs }}=l_{t}+a
$$

and

$$
\Delta \mathrm{l}_{2}=\mathrm{a} \text {. }
$$

An error of the zero arises, for example, due to a shift in the origin of coordinates in the course of a dimensional measurement.

b. Error of the Unit: Errors of the unit occur for measurement systems which - while having a properly linear relation of measured value to true value have a non-unitary slope, that is:

$$
l_{\text {obs }}=(1+b) \cdot \ell_{t}
$$

and

$$
\Delta l_{\mathrm{g}}=\mathrm{b} \cdot \mathrm{l}_{\mathrm{t}}
$$

Error of the unit corresponds, for example, to an erroneous value of wavelength in displacement interferometry or a constant misalignment of coordinate and object axes.

c. Error of the Scale: Errors of the scale occur for measurement systems which have a non-linear relation of measured value to true value, that is:

$$
\Delta \ell_{l}=c\left(l_{t}^{n}\right) \text {. }
$$


Error of the scale represents failure of the interpolation scheme to divide the difference between the unit and the zero into strictly equal intervals, that is, the degree to which the system is nonlinear. The measurement system is linear, if given four objects (i.e., four displacements, positions, distances or extensions) for which the true difference between pairs is the same, the measured differences are also the same. Error of the scale is, by definition, the inequality in those differences, that is:

$$
\Delta_{\mathrm{t}}=\left(l_{\mathrm{obs} 1}-l_{\mathrm{obb2} 2}\right)-\left(l_{\mathrm{obb3} 3}-l_{\mathrm{obs} 4}\right)
$$

when

$$
\left(l_{11}-l_{2}\right)=\left(l_{13}-l_{12}\right) \text {. }
$$

That the error of the scale corresponds to a non-zero value of the coefficient $c\left(\ell_{t}^{p}\right)$ can be seen when the non-linear response of a dimensional measurement system is given a specific form, such as the quadratic:

$$
\ell_{\mathrm{obs}}=\mathrm{a}+(1+\mathrm{b}) \cdot \ell_{\mathrm{t}}+\mathrm{cl}_{\mathrm{t}}^{2} .
$$

Evaluation of Eq.30 at four values of $\ell_{v}$ denoting the differences in the pairs of true values by $\Delta_{\mathfrak{i}}$ and substitution into Eq. 26 leads to the result:

$$
\Delta \ell_{\mathrm{b}}=\mathrm{c} \cdot\left(\ell_{11}-\ell_{\mathrm{B}}\right) \cdot \Delta_{\mathrm{i}} .
$$

An error of the scale arises, for example, from to faulty interpolation of fringes in interferometry or uncompensated nonlinearities in LVDT or capacitive displacement measuring devices.

d. Errors of Zero, Unit and Scale: In summary, the axiom-specific errors for the general case that $\ell_{o b s}=a+(1+b) \cdot \ell_{t}+c\left(\ell_{t}^{2}\right)$ are given by:

$$
\Delta \ell=\Delta \ell_{2}+\Delta l_{\mathrm{b}}+\Delta \ell_{\mathrm{l}}=\mathrm{a}+\mathrm{b} \cdot \mathrm{l}_{\mathrm{t}}+\mathrm{c}\left(\mathrm{l}_{\mathrm{t}}^{\mathrm{a}}\right)
$$

where: a corresponds to $\Delta \ell_{2}$, the error in realizing the zero; $b$ corresponds to $\Delta \ell_{w}$, the error in realizing the unit; $c$ corresponds to $\Delta \ell_{\text {, }}$, the error in realizing the scale; and $\ell$ is any one of the four dimensional measurement types.

\section{PRINCIPAL SPECIFIC SOURCES OF ERRORS}

Errors in dimensional measurements are particular to each of the different types and, since each succeeding type is dependent on that which precedes it, total error increases as one proceeds from the displacement, position and distance to the extension of objects. 


\section{A. Error in Displacement Interferometry}

The type of dimensional measurement most directly linked to the SI unit of length and the basis for all subsequent types of dimensional measurements is displacement by laser interferometry, that is, the measurement of a linear change of location in space with time of a single object, in this case, the moving mirror of the interferometer. The measure of the limit of this ability is displacement uncertainty or error, $8 \mathrm{D}$. Errors in measurement of displacement by laser interferometry are associated with each of the terms in Eq. 15.

\section{Error of Wavelength in Vacuum $\lambda_{0}$}

The uncertainty in the wavelength in vacuum of the laser light depends on the type of stabilization used in its construction. As indicated in Table 3 above, uncertainty in the vacuum wavelength of the $633-\mathrm{nm}$ red-orange line of the helium-neon $(\mathrm{HeNe})$ lasers widely used in dimensional metrology range from $10^{-6}$ for unstabilized lasers down to $10^{-10}$ for iodine-stabilized ones.

\section{Error of Index of Refraction of Medium n}

The uncertainty in the wavelength in medium of the laser light depends on the index of refraction of the medium through which the light propagating in the interferometer passes.

a. Error of the Index of Ambient Air: Since index of refraction of a gas is a function of its temperature, pressure, humidity and composition, without compensation for actual variations in those parameters the upper limit of uncertainty for interferometric displacement measurements in ordinary ambient air can be large: for example, an index error of $10^{-6}$ would result from any one of the following variations: a one degree Centigrade change in temperature, a $2.5 \mathrm{~mm} \mathrm{Hg}$ change in atmospheric pressure, or an $80 \%$ change in relative humidity [13].

b. Error of the Index of Compensated Standard Air. Errors associated with the index of refraction of the ambient medium can be reduced by compensation achieved by measurement of the index of the actual ambient air or by calculation of the index of standard dry air. The Edlen formula is an internationally agreed upon equation for the calculation of the index of refraction of standard air as a function of wavelength, air temperature, air pressure and relative humidity [14].

With compensation, the lower limit of uncertainty for practical laser displacement interferometry in ambient air is estimated to be about $1.2 \cdot 10^{-7}$ 
[15]. The uncertainty in the Edlen formula itself for the index of refraction of standard dry air is estimated to be $5 \cdot 10^{-8}[16]$. Operation of an interferometer in vacuum eliminates this source of error.

c. Error of Index of Refraction in the Dead Path: So-called "dead path" error is due to improper compensation for the difference in the lengths of the optical paths of the two interfering beams at the zero-displacement position of the system when index-altering environmental changes occur during the course of the displacement measurement. For commercial systems which use sensorbased index compensation, dead-path errors are largely compensated for in software, leaving a residual error of $1.4 \cdot 10^{-7}$ times the dead-path distance.

\section{Error in Fringe-Fractioning}

Errors in realizing a scale by means of displacement interferometry arise from various sources in the process of the generation, subdivision and counting of the interference fringes. Representative of such errors are those which arise in widely-used displacement interferometers of the polarization beam-splitting type. The principal sources of error in this type of system are those associated with electronic subdivision, polarization mixing, thermal drift and dead path [17].

a. Error in Electronic Subdivision: The inherent half-wavelength resolution of interferometers, corresponding to the spacing of the alternating light and dark of the fringes, can be extended by a variety of electronic fringe-interpolation schemes, each of which has its own limiting resolution which contributes an additive, least-count error. Operating at $633 \mathrm{~nm}$, certain commercial polarization heterodyne interferometer systems have least counts in electronicsubdivision, depending on the whether used with retro-reflectors or plane mirrors, of $\lambda / 32$ (approximately $20 \mathrm{~nm}$ ) and $\lambda / 64$ (approximately $10 \mathrm{~nm}$ ) respectively.

b. Error from Polarization Mixing: Less-than-perfect separation of the polarization states of the interfering beams in polarization-type interferometers, due to leakage of one component into the other, produces a non-linear error in displacement measurement with such systems. Such error varies as a function of change in optical path with a periodicity of the wavelength of the laser source with an amplitude specific to an individual interferometers. For one commercial linear interferometer system, the peak-to-peak phase error was found to be $5.4^{\circ}$ corresponding to approximately $5 \mathrm{~nm}$. 
c. Error from Thermal Drift: Changes in temperature within the optical components of the interferometer system, which produce a differential change in path optical length between the interfering beams, give rise to drift type errors in displacement measurement. This environmentally induced error is less for temperature-controlled systems and those which are specifically designed to deal with this source of error. An example of the latter has a quoted thermal error of $40 \mathrm{~nm} / \mathrm{C}^{\circ}$, twelve times better than a conventional plane-mirror system with its $0.5 \mu \mathrm{m} / \mathrm{C}^{\circ}[12]$.

\section{Error in Alignment of Interferometer}

Finally, constant angular misalignment between the interferometer and the incident light gives rise to an error (derived from the basic interferometer equation):

$$
8 D_{\theta}=D \cdot\left(-\theta^{2} / 2\right),
$$

where $8 D_{\theta}$ is misalignment contribution to the displacement error, $D$ is the measured displacement, and $\delta \theta$ is the small angle of misalignment between the axis of interferometer (the normal to the parallel faces of the interferometer mirrors) and the axis of propogation of the incident laser light.

In sum, this section has looked at sources of errors in the type of dimensional measurement most directly linked to the SI unit of length, those of laser displacement interferometry by the best available form of that technique, polarization-type heterodyne interferometry.

\section{B. Error in Coordinate-Position Measurement}

After displacement, the type of dimensional measurement next most directly linked to the SI unit of length and inherent in subsequent types is position, that is, measurement of the location of a single point relative to a coordinate system. The measure of the limit of the ability to measure position is position error, $\mathbf{8 P}$. Errors in position measurements are associated with each of the functional components of the coordinate measurement system as embodied by a coordinate measuring machine: the scales, frame, carriage and probe. 


\section{Error In Relation to Displacement Scales}

Lack of commonality of origin and co-linearity of axes of the coordinateposition measurement system and the displacement system, which supplies the metric and scale subdivisions, gives rise to two types of errors specific to positional measurement.

a. Misalignment of Mirror Translation: Constant angular misalignment between the axis of the displacement measurement and the axis of the coordinate system gives rise to "cosine error," which is governed by the relationship:

$$
\mathrm{P}=\mathrm{D} / \cos \alpha
$$

where $\mathrm{D}$ is the displacement, $\mathrm{P}$ is the coordinate position being assigned by the measurement and $\alpha$ is the angle of misalignment between the two. For a displacement generated by an interferometer system, $\alpha$ is the angle between the axis of translation of the moving mirror and the optical axis of the interferometer (i.e., the normal to the parallel faces of the reference and moving mirrors). For a small angle, the cosine error contribution to coordinate-position error, a multiplicative error, is given by:

$$
\delta P_{c}=D \cdot\left(-\alpha^{2} / 2\right) .
$$

Cosine error arises, in effect, when the axis of the displacement vector and axis of the coordinate vector are rotated with respect to each other but have a common origin. [Note that the errors due respectivelt to misalignment of interferometer and light (Eq.34) and misalignment of mirror translation and interferometer (Eq.36) are of the same sign while that due to misalignment of object and mirror translation (Eq.40) is opposite (Appendix A)].

b. Abbe Error: Abbe error can arise when the axes of the displacement vector and the coordinate vector do not share a common origin and they rotate with respect to each other, that is, tilt, during the course of the displacement. Abbe error is governed by the relationship:

$$
\mathrm{D}=\mathrm{P}+\mathrm{O}_{2} \cdot \sin \phi
$$

where $\mathrm{D}$ is the measured displacement, $\mathrm{P}$ is the coordinate being assigned by the measurement, $\mathrm{O}_{2}$ is the lateral distance between the displacement and coordinate axes (called the Abbe offset), and $\phi$ is the angle of tilt which occurs during the course of the displacement measurement. For small angles, the 
Abbe error contribution to coordinate-position error, which is an additive, is given by:

$$
\delta \mathrm{P}_{2}=\mathrm{O}_{\mathrm{a}} \cdot \phi .
$$

\section{Error Related to Carriage Motion}

Carriage-associated position-measurement error occurs in measuring machines in which the motion-generating system (which translates the object relative to the reference frame) is the same as the displacement-measuring system (which measures that translation) as, for example, in a machine which uses the same lead screw both to move a carriage and to measure its position. For such machines, carriage-associated errors include backlash and hysteresis, both of which are direction-dependent and result in loss of origin of the coordinate system.

\section{Errors Related to Reference-Frame Geometry}

Limitations in the mechanical structure of a coordinate measuring machine give rise to positional errors associated with the machine's coordinate axes and their angular relationships. Since the reference frame of a coordinate measuring machine must consist of one highly-planar reference surface for each coordinate axis with each of these planes angularly oriented with respect to the others in a constant and well known way, the errors associated with reference frame geometry are complex.

Characterization of the positional errors in a 3D CMM requires dealing with twenty-one degrees of freedom based on six degrees per axis (three translational plus three angular, including roll, pitch and yaw) plus the three angular orientations of the axes with respect to each other. Specialized matrixbased models and measurement algorithms for characterizing positional errors in coordinate measuring machines and machines tools have been developed $[18,19]$ and standardized techniques adopted [20].

\section{Error Related to the Probe}

Limitations in the mechanism for linking the object to be measured to the machine's coordinate measurement system give rise to positional errors specifically associated with the machine's probe. Probe-specific errors are of two types, depending on whether the probe is designed to carry out only functions of a probe as a simple sensor or a probe as a measuring machine.

a. Positional Error of Probe-as-Sensor: Positional errors of a probe-as-sensor arise to the degree that it fails to carry out its primary function, that of 
detecting -- in a binary sense of "here" or "not here" -- the boundary of an object, such as to locate it relative to the coordinate system of the machine. Positional errors of probe-as-sensor include what in touch-trigger mechanicalcontact probes is called "pre-travel," that is, the displacement of the probe tip after contact with the object but before the probe signals that contact. Such error arises, for example, from variations in bending of the probe structure and the threshold response of the mechanical-to-electrical transducer.

b. Positional Error of Probe-as-Measuring-Machine: Positional errors of a probe-as-measuring-machine arise in, for example, "analog probes," which are designed not only to carry out the primary function of linking machine and object, but also to provide an output signal proportional to the displacement of the probe tip on contact with the object. Positional errors of analog probes, being as they are miniature measuring machines attached to a larger machine include: 1) errors of the probe as a sensor; 2) errors of the probe as a measuring machine (including those associated with scale, frame, and carriage); and 3) errors associated with the orientation of the probe as one coordinate system with respect to another.

In sum, this section has looked at sources of errors in measurements of position, which after displacement, is the next most directly linked to the SI unit of length. As indicated, position error deals with the ability to measure location of points in space and are associated with each of the functional components of a coordinate measuring machine by which such position measurements are made.

\section{Error in Distance Measurement}

After displacement and position, the type of dimensional measurement next most directly linked to the SI unit of length is that of distance, that is, measurement of the separation of two successively located point-like features on material objects. The measure of the limit of the ability to measure distance is distance error, $\mathbf{8 d}$. Errors specific to measurements of distance are associated with the probing, angular alignment and environmental-dependence of separation of distance-type features of the object. 


\section{Distance Error from Feature Probing}

Errors of feature probing arise from variations in the size, shape and material of the individual point-, line- and plane-like features, the separation of which is being measured in a distance measurement. Such variations limit the ability to reproducibly locate features with a probe. Errors associated with such variations are assessed in terms of the repeatability with which the probe of a particular coordinate measuring machine can be set on a particular feature.

\section{Misalignment of Object and Coordinate Axes}

Misalignment between the object being measured and a coordinate axis of the measuring machine gives rise to cosine error governed by the relationship:

$$
d=\left|P_{1}-P_{2}\right| \cdot \cos \gamma,
$$

where $P_{1}$ and $P_{2}$ are the measured coordinate positions of the point-like features, $d$ is the distance being measured and $\gamma$ is the angle of misalignment between the coordinate axis and that of the object itself. For a small angle, the cosine error contribution to distance error is given by:

$$
\delta d_{c}=d \cdot\left(+\gamma^{2} / 2\right) .
$$

\section{Error from Object Environment}

Apart from the effects of changes in the environment on displacementmeasuring devices and the measuring machine itself, such environmental changes give rise to errors associated with the object itself, including those associated with thermal expansion, mechanical distortion and contamination.

a. Error from Object Thermal-Expansion: Distance error due to thermal expansion of the object can be due to different temperatures for the whole object or different temperatures for two positions on the object. In either case, variation in the dimensions of the object with temperature are governed by the equation:

$$
\rho=-\left(\Delta \ell / \ell_{0}\right) / \Delta T,
$$

where $\rho$ is the coefficient of linear thermal expansion of the material and $\Delta / / \ell_{0}$ is the fractional change in the "length" of material for a difference in temperature $\Delta \mathrm{T}$. Note that since thermal expansion is defined in terms of the 
change in length (either between distance-type features or extension-type boundaries), it is inherently a distance-type property given by:

$$
\delta \mathrm{d}_{\mathrm{T}}=\rho \cdot \Delta \mathrm{T} .
$$

b. Error from Object Distortion: Distance error can also arise from any other factor which, in effect, distorts the object by altering the relative separation of features of the object. Such factors include any local or overall mechanical distortion of the object due, for example, to fixturing of the object on the measuring machine; and any gain or loss of material on one feature with respect to the second due, for example, to contamination or wear.

In sum, this section has looked at sources of errors in measurements of distance is, which after displacement and position, the next most directly linked to the SI unit of length, such errors being associated with measurement of the separation of two point-like features of material objects.

\section{Error in Extension Measurement}

The type of dimensional measurements operationally farthest removed from the primary realization of the SI unit of length is that of extension. The measure of the limit of the ability to measure extension is extension error, $\delta$ e. Most specific to the measurement of extension-type features of objects, such as the inside diameters of holes and the outside diameters of plugs, is error associated with locating a single boundary and error associated with locating one boundary relative to another one.

\section{Error in Location of a Single Boundary}

a. Error in Reducing a 3D Surface to a Point: Errors occur in measuring the location of a real boundary because a material object is not an ideal geometrical surface being intersected by a line such as to define a point. Instead that boundary is a rough three-dimensional surface being contacted by a finite-area probe. Estimates of the contribution to boundary-location errors for specific object-probe combinations may be made based on measurements of surface roughness, which involves statistical averaging over surface irregularities. Boundary-location errors due to surface roughness can be comparable to 
the roughness itself, which for a highly-polished steel surface, for example, can be of the order of $0.05 \mu \mathrm{m}$.

b. Error in Compensation of Probe-Object Interaction: Errors occur in measuring the position of a single boundary as a result of any uncompensated off-set between the location of the probe and the location of the surface which the probe signals. For example, in locating the boundary of a machined part with a mechanical stylus, single-boundary-location errors occur due to incomplete compensation for both: the finite radius of the probe and its finite penetration into the part. Analogous errors occur in locating the boundary of a microcircuit element with the beam of an electron microscope.

Compensation for probe-object interaction, such as the deformation of a part by a mechanical stylus and penetration into the bulk of a material by an electron beam, can only be done by fundamental theoretical modelling of the specific probe and object involved. Of necessity, such modelling has been done, for example, for high-accuracy optical- and electron-microscope calibrations of photomask linewidth standards for use in the microelectronics industry [21].

In sum, error in boundary location is a vector having a magnitude (associated with, for example, the finite roughness of the surface, the finite extent of the probe and the finite depth of penetration of the object by the probe) and a direction, positive or negative, relative to the axis of coordinate measurement.

\section{Error in Location of Two Boundaries}

In measurements of extension, errors in the location of one boundary combine as vectors with errors in the location of the second boundary - that is, one of opposite orientation - and the results are non-canceling, as indicated by:

$$
\delta_{e}=8 P_{b 1}+8 P_{b 2}
$$

where $\delta \mathrm{P}_{\mathrm{b} 1}$ is the error in the location of the first boundary and $\delta \mathrm{P}_{\mathrm{b} 2}$ is the error in the location of the second.

For the case that the boundary-location error is the same for the two boundaries, that is, where the magnitude of $\delta \mathrm{P}_{\mathrm{b} 1}$ equals that of $\delta \mathrm{P}_{\mathrm{b} 2}$, the error in extension measurement due to the combination of the two boundary-location errors is:

$$
\delta_{e}=\mp 28 P_{b}
$$


where the minus sign is for outside-caliper measurements (as in the diameter of a plug) and the plus sign is for inside-caliper measurements (as in the diameter of a hole) when the boundary-location errors are due, in effect, to "penetration" (positive or negative) of the object by the probe.

An example of a positive boundary-location error is that due to the finite radius of a mechanical probe (that is, the extension of the probe tip along the axis of measurement). An example of a negative boundary-location error is probe pre-travel (that is, the finite displacement of the probe along the axis of measurement after mechanical contact with the object, but before the probe transducer crosses a threshold and signals).

Additive boundary-location errors are unique to extension-type measurements. In measurements of displacement, position and distance, the boundaries involved face in the same direction and boundary-location errors cancel, that is:

$$
8 \mathrm{P}_{\mathrm{D}, \mathrm{P}, \text { or d }}=8 \mathrm{P}_{\mathrm{b}}-8 \mathrm{P}_{\mathrm{b}} \equiv 0 .
$$

\section{E. Compounding of Error in Measurement Types}

A final aspect of assessing accuracy in practical dimensional measurements involves a look at the cascading nature of the errors in the succession of the dimensional measurement types. This cascading, that is, accumulation, of errors is shown formally below, where $\Delta$ represents the total uncertainty of the type of measurement and $\delta$ represents the type-specific error in that total.

In this notation, the total error in displacement measurement $\Delta \mathrm{D}$ a function of $8 \mathrm{D}$, which is all the displacement-specific errors described in Section IVA, plus $\Delta \lambda_{0}$, which is the error specific to the metric-carrying vacuum wavelength of laser light source, and is given by:

$$
\Delta \mathrm{D}=\Delta \mathrm{D}\left(8 \mathrm{D}, 8 \lambda_{0}\right) .
$$

Similarly, the total error in position measurement $\Delta p$ a function of $8 p$, which is all the position-specific errors described in Section IVB, plus $\Delta \mathrm{D}$, which is the total error in displacement measurement, and is given by:

$$
\begin{aligned}
\Delta p & =\Delta p(8 p, \Delta D) \\
& =\Delta p\left(\delta p, 8 D, 8 \lambda_{0}\right) .
\end{aligned}
$$

And again, the total error in distance measurement, $\Delta d$, a function of the total error in position measurement, plus all the distance-specific errors described in Section IVC above, and is given by: 


$$
\begin{aligned}
\Delta \mathrm{d} & =\Delta \mathrm{d}(\delta \mathrm{d}, \Delta \mathrm{p}) \\
& =\Delta \mathrm{d}\left(\delta \mathrm{d}, \delta \mathrm{p}, \delta \mathrm{D}, \delta \lambda_{\mathrm{o}}\right)
\end{aligned}
$$

Finally, the total error in extension measurement, $\Delta e$, a function of the total error in distance measurement, plus the extension-specific errors described in Section IVD above, and is given by:

$$
\begin{aligned}
\Delta \mathrm{e} & =\Delta \mathrm{e}(\delta \mathrm{e}, \Delta \mathrm{d}) \\
& =\Delta \mathrm{e}\left(\delta \mathrm{e}, \delta \mathrm{d}, \delta \mathrm{p}, \delta \mathrm{D}, \delta \lambda_{\circ}\right)
\end{aligned}
$$

As is indicated by this formal analysis, the accuracy of each type of dimensional measurement depends on - and is limited by - the total error of the preceding type, as well as by the sum of its own type-specific errors.

In sum, measurements of extension are the most difficult in which to achieve accuracy because they inherit all the errors of the successive displacement, location and distance measurements upon which they are based and, addition, are subject to intractable boundary-location errors to which the others are not.

\section{ERROR-BUDGET EXAMPLE}

All of the considerations of accuracy assessment discussed above can be taken into systematic account in the assessment of the errors in a particular dimensional measurement system by compilation of an error budget for the measuring system in question. In this example, a simple sum-over-errors is used to estimate the total worst-case error; other means for estimating the most likely errors have been described [22].

The example considered here is measurement of an extension-type object on a laser-interferometer-based single-axis coordinate measuring machine. In this example, the overall system a function of a stabilized $\mathrm{HeNe}$ laser, a commercial heterodyne interferometer system, a machine frame with a moving carriage, a touch-fire probe, and a $100 \mathrm{~mm}$-long steel rod, the length of which is to be measured, all within a normal atmospheric environment.

Table 6 below shows a compilation of the contributions to the overall accuracy of measurement of the specified object with the hypothetical coordinate-measuring-machine system described. The organization of the error budget follows the sequence of the type scheme used throughout the chapter. 
In the table, $\delta \ell_{\mathrm{Ai}}$ and $\delta \ell_{\mathrm{Bi}}$ represent, respectively, additive and multiplicative contributions to the type-specific errors. The values are estimates of the relative size of error contributions from the various sources. In the following sections, each of the individual contributions to the type-specific errors for this hypothetical example are discussed in turn.

\section{A. Displacement Errors}

In this example the errors in the laser-interferometric measurement of the displacement derive from displacement-specific errors associated with each of the parameters in the basic interferometer equation plus the error associated with the vacuum wavelength of the laser light. 
Table 6. Error-Budget Example: An Analysis of a Measurement of the Extension of a $100 \mathrm{~mm}$-Long Object with an Interferometer-Based Coordinate Measuring Machine

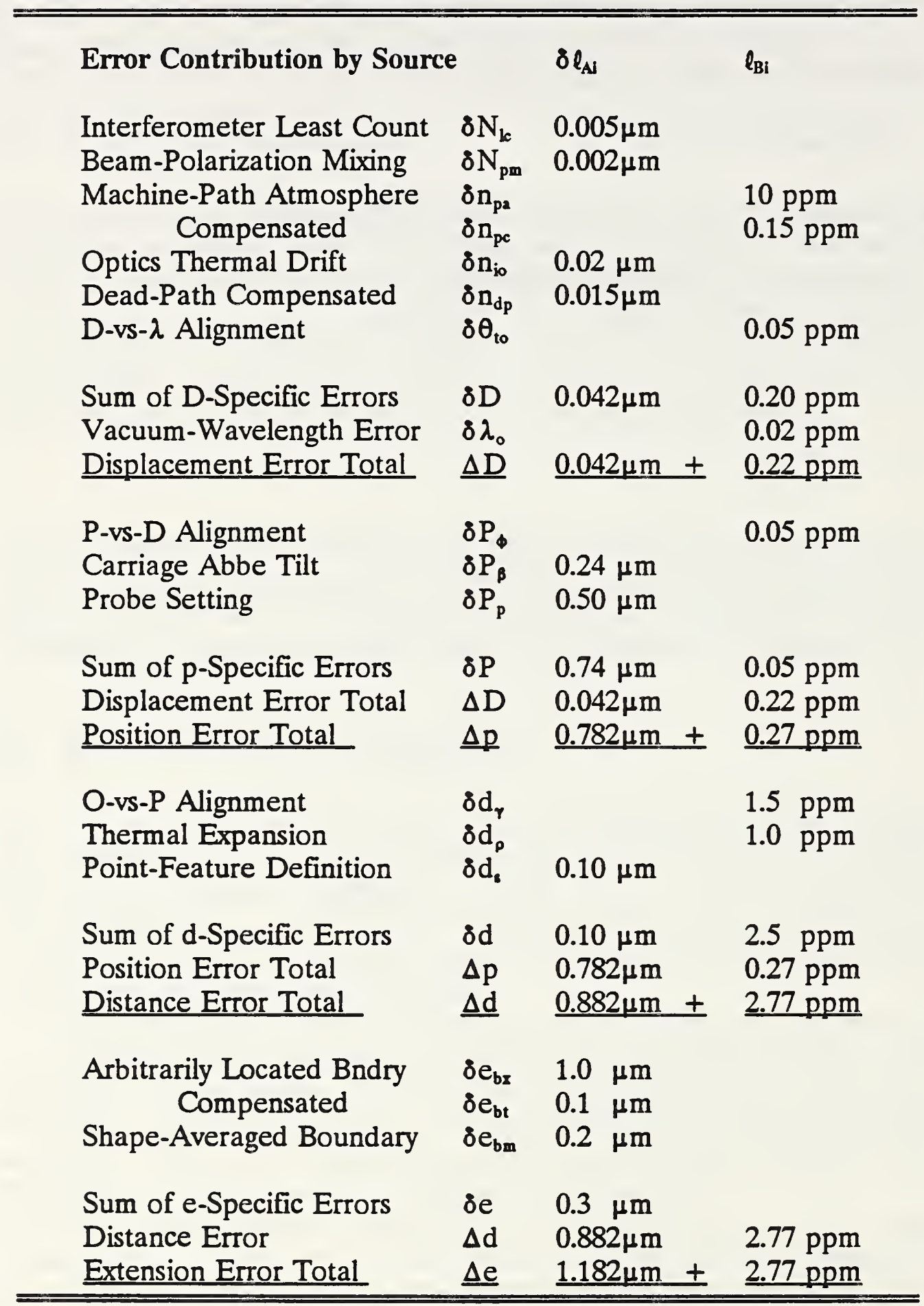




\section{Fringe-Fraction Error $\delta D_{N}$}

The contributions to displacement-specific measurement error are due to errors in determination of the change in the order of interference with the translation of the interferometer reflector:

$$
N=i+f
$$

where $\mathrm{N}$ is a real number which is the sum of the counted order $\mathrm{i}$ and measured fringe fraction $\mathrm{f}$. Practically, the total error in $\mathrm{N}$ is associated with error in interpolating between fringes, that is:

$$
8 \mathrm{~N}=8 \mathrm{f} .
$$

In this example, the contributors to error in fringe-fractioning are the $5 \mathrm{~nm}$ due to the least count of interferometer and the $2 \mathrm{~nm}$ due to the mixing of the polarization states of the interfering beams.

\section{Index-of-Refraction Error $8 D_{n}$}

Contributions to displacement-specific measurement error arise from variations in the indices of refraction along the optical paths of the interfering beams due to changes in environmental conditions. The variation in phase between the two beams as a function of variations in the individual indices of refractions in each of the segments of the reference- and moving-arm beams is given by:

$$
\delta \Delta \phi=\left(2 \pi / \lambda_{0}\right)\left[\Sigma_{\mathrm{i}} \delta \mathrm{n}_{\mathrm{ri}} \cdot \ell_{\mathrm{ri}}-\Sigma_{\mathrm{i}} \delta \mathrm{n}_{\mathrm{mi}} \cdot \ell_{\mathrm{mi}}\right],
$$

where $\Sigma_{i}$ indicates a sum over the i number of optical-path segments $n_{i} \cdot \ell_{i}$ in each of the arms. In this example, the principal segments of the overall optical path include the CMM machine-axis path along which the moveable reflector. is translated, the dead path, and the interferometer-optics path, that is, the glass through which the light passes.

a. Machine-Path Atmosphere: The first and major source of index-of-refraction errors is the variation of the atmospheric air along the displacement path. The following show the changes in temperature, pressure, and relative humidity each of which gives rise to a displacement error of $10^{-6}$ :

$$
\begin{aligned}
& 8 \mathrm{n}_{\Delta \mathrm{T}}=1 \mathrm{ppm} / 1^{\circ} \mathrm{C} \\
& 8 \mathrm{n}_{\Delta \mathrm{P}}=1 \mathrm{ppm} / 2.5 \mathrm{~mm} \mathrm{Hg} \\
& \delta \mathrm{n}_{\Delta \mathrm{H}}=1 \mathrm{ppm} / 80 \% \Delta \mathrm{RH} .
\end{aligned}
$$


In this example, a $\pm 0.5^{\circ} \mathrm{C}$ change in temperature, a $\pm 25 \mathrm{~mm}$ change in pressure, and a $\pm 10 \%$ change in relative humidity combine to produce a multiplicative error of about $10 \mathrm{ppm}$. With measurement of the changes and compensation for them, this is reduced to $0.15 \mathrm{ppm}$.

b. Interferometer-Optics Thermal Drift: The second source of index of refraction error is change in the temperature of the glass components of the interferometer through which the light in the two beams passes. In this example, optics thermal drift error is $0.02 \mu \mathrm{m}$.

c. Dead-Path Compensation: A last source of index of refraction error is change in temperature, pressure, and humidity of the dead-path (the difference in the interferometer arms at the starting point of the machine-axis path). In this example, compensation has reduced dead-path error to $0.015 \mu \mathrm{m}$.

\section{Interferometer-Axis Alignment Error $8 \mathrm{D}_{\theta t i}$}

The contribution to displacement-specific measurement error due to the angular misalignment $\theta_{\mathrm{t}}$ between the axis of the interferometer and the axis of propagation of the incident light is, for a small angle of misalignment, given by:

$$
\delta D_{\theta t i}=D \cdot\left(-\theta_{t i}^{2} / 2\right) \text {. }
$$

For an angular alignment corresponding to a lateral displacement of one-tenth the spot size of a $3 \mathrm{~mm}$-beam at the one-meter travel of the measuring machine stage, $\theta_{t \mathfrak{t}}=3 \times 10^{-3}$ radians and the resulting error $8 D_{\theta i}$ is $0.05 \mathrm{ppm}$.

\section{Sum of Displacement-Specific Errors 8D}

In this example, the total displacement-specific error $8 D$ is the sum of the errors due to fringe-fractioning, index of refraction, and angular misalignment of translation-propagation axes and is given by:

$$
\begin{aligned}
8 \mathrm{D} & =\Sigma_{\mathrm{i}} 8 \mathrm{D}_{\mathrm{i}} \\
& =\left(\Sigma 8 \mathrm{D}_{\mathrm{Ai}}\right)+\left(\Sigma \delta \mathrm{D}_{\mathrm{Bi}}\right) \cdot \ell \\
& =0.042 \mu \mathrm{m}+0.20 \mathrm{ppm} \cdot \mathrm{D} .
\end{aligned}
$$

At a displacement of $100 \mathrm{~mm}$ (corresponding to the extension of the object being measured), the sum of the displacement-specific errors is: 


$$
\delta \mathrm{D}_{100}=0.062 \mu \mathrm{m}=0.62 \mathrm{ppm} @ 100 \mathrm{~mm}
$$

\section{Vacuum-Wavelength Error $\delta \lambda_{0}$}

The contribution to the total displacement measurement error $\Delta \mathrm{D}$ due to the error in the value of the vacuum wavelength of the laser is:

$$
\delta D_{\lambda_{0}}=\left(\delta \lambda_{0} / \lambda_{0}\right) \cdot D,
$$

which for the highly stabilized commercial laser of this example is $2 \cdot 10^{-8}$. Thus,

$$
\delta \mathrm{D}_{\lambda_{0}}=0.02 \mathrm{ppm} \cdot \mathrm{D} \text {. }
$$

\section{Total Displacement Error $\Delta \mathrm{D}\left(\delta \mathrm{D}, \delta \lambda_{0}\right)$}

As indicated, combining the displacement-specific error $\delta \mathrm{D}$ with the error in the vacuum-wavelength of the laser source $\delta \lambda_{0}$ yields the total displacement error $\Delta \mathrm{D}$ over the one-meter travel of the measuring machine stage, that is:

$$
\begin{aligned}
\Delta \mathrm{D} & =\delta \mathrm{D}+\delta \lambda_{0} \\
& =\left(\Sigma \delta \mathrm{D}_{\mathrm{Ai}}\right)+\left(\Sigma \delta \mathrm{D}_{\mathrm{Bi}}\right) \cdot \ell+\left(\delta \lambda_{\mathrm{o}} / \lambda_{0}\right) \cdot \ell \\
& =0.042 \mu \mathrm{m}+0.22 \mathrm{ppm} \cdot \mathrm{D}
\end{aligned}
$$

For a displacement measurement corresponding to length of the 100 -mm-long object, the total error in the displacement measurement is:

$$
\Delta \mathrm{D}=0.064 \mu \mathrm{m}=0.64 \mathrm{ppm} @ 100 \mathrm{~mm} .
$$

\section{B. Error in Position Measurement $\Delta \mathbf{P}$}

In this example the errors in the coordinate-measuring-machine measurement of position derive from errors associated with the geometrical relationships among the scale, frame, carriage and probe of the CMM and the setting ability of the probe itself.

\section{Misalignment of Coordinate and Displacement}

The contribution to position-specific measurement error due to the angular misalignment $\phi$ between the axis of translation of the mirror and the axis of the interferometer is, for a small angle, given by: 


$$
\delta \mathrm{P}_{\phi}=\mathrm{D} \cdot\left(-\phi^{2} / 2\right) .
$$

For an angular misalignment of $\phi_{\mathrm{ti}}=3 \cdot 10^{-3}$ radians, the resulting error is:

$$
8 \mathrm{P}_{\phi}=0.05 \mathrm{ppm} \cdot \mathrm{P} .
$$

\section{Carriage Abbe Tilt}

The contribution to position-specific measurement error due to a difference in angle of tilt of a non-zero-length probe between two positions (Abbe error) is, for a small angle:

$$
\delta \mathrm{P}_{\mathbf{a}}=\mathrm{O}_{\mathrm{a}} \cdot \beta .
$$

In this example, for a distance between the axis of the probe and the axis of the displacement measurement of $50 \mathrm{~mm}$ and for a change in tilt of $1 \mathrm{arc}$ second, the Abbe error contribution to coordinate-position error, which is an additive, is:

$$
\delta \mathrm{P}_{2}=0.24 \mu \mathrm{m} .
$$

\section{Probe Setting}

The contribution to position-specific measurement error due to probe setting is a measure of the ability of an individual probe to reproducibly locate unvarying, uni-directionally approached, probed features. Since a location measurement requires the probe to trigger twice (once at the location of the origin and once at the location being measured, an estimate of the error in probe-setting based on the $2 \sigma$ setting of a good-quality commercial probe operated in the touch-trigger mode is:

$$
\delta \mathrm{P}_{\mathrm{p}}=2 \cdot 0.25 \mu \mathrm{m}=0.50 \mu \mathrm{m} .
$$

\section{Sum of Position-Specific Errors 8p}

In this example, the total position-specific error $\delta \mathrm{P}$ is the sum of the errors due to coordinate-scale misalignment, Abbe error, and probe setting.

$$
\begin{aligned}
\delta \mathrm{P} & =\Sigma \delta \mathrm{P}_{\mathrm{i}} \\
& =0.74 \mu \mathrm{m}+0.05 \mathrm{ppm} \cdot \mathrm{d} \\
& =0.745 \mu \mathrm{m} @ 100 \mathrm{~mm}
\end{aligned}
$$




\section{Total Position Error $\Delta P(8 P, \Delta D)$}

Combining the position-specific errors, $\delta$, with the total displacement error, $\Delta \mathrm{D}$, over the one-meter travel of the measuring machine stage yields:

$$
\Delta \mathrm{P}=\delta \mathrm{P}+\Delta \mathrm{D}=0.782 \mu \mathrm{m}+0.27 \mathrm{ppm} \cdot \mathrm{P} .
$$

For a position corresponding to length of the $100 \mathrm{~mm}$-long object, the total error in the position measurement is:

$$
\Delta \mathrm{P}_{100}=0.809 \mu \mathrm{m}=8.1 \mathrm{ppm} @ 100 \mathrm{~mm}
$$

\section{Error in Distance Measurement $\Delta d$}

In this example the errors in the coordinate-measuring-machine measurement of distance derive from distance-specific errors associated with the object alignment, temperature and distance-type features, plus the error in position measurement.

\section{Object-Axis/Probe-Path Alignment}

The contribution to distance-specific error due to the angular misalignment $\gamma$ between the axis of the object and the axis of coordinate measurement, that is, the path of the probe, is, a small angle, given by:

$$
\delta d_{\gamma}=d \cdot\left(+\gamma^{2} / 2\right) \text {. }
$$

For an angular misalignment of $0.1^{\circ}$ (corresponding to a lateral distance of less than $0.2 \mathrm{~mm}$ over the $100 \mathrm{~mm}$ length of the object):

$$
\delta \mathrm{d}_{\mathrm{\gamma}}=1.5 \mathrm{ppm} \cdot \mathrm{d} .
$$

Note that this error due to misalignment of object and coordinate axis (Eq.40) is opposite in sign to that due to misalignment of interferometer and light (Eq.34) and misalignment of mirror translation and interferometer (Eq. 36).

\section{Object Thermal Expansion}

The contribution to distance-specific error due to the difference in temperature $\Delta \mathrm{T}$ between the object to be measured and a reference temperature (such as that of the CMM) is determined by the linear thermal expansion coefficient $\rho$ for the object according to the thermal expansion equation: 


$$
8 \mathrm{~d}_{\Delta \mathrm{T}}=\rho \cdot \Delta \mathrm{T} \cdot \mathrm{d}
$$

For the a steel object in this example, $\rho$ is $10 \mathrm{ppm} /{ }^{\circ} \mathrm{C}$, which for temperature difference $\Delta \mathrm{T}$ of $0.1^{\circ} \mathrm{C}$ gives:

$$
8 \mathrm{~d}_{\Delta \mathrm{T}}=1.0 \mathrm{ppm} \times \mathrm{d} .
$$

\section{Object Point Definition}

The contribution to distance-specific measurement error due to object point definition is a measure of the ability to associate with the object features which can be characterized as points, the position of which points can be reproducibly located by a variability-free probe. In this example, the estimate of a practical lower limit for error associated with locating points on real objects (twice that for defining a single point) is estimated to be:

$$
\delta \mathrm{d}_{\mathrm{s}}=0.10 \mu \mathrm{m} .
$$

\section{Sum of Distance-Specific Errors 8d}

In this example, the total distance-specific error $8 D$ is the sum of the errors due to coordinate-scale misalignment, Abbe error, and probe setting.

$$
\delta \mathrm{d}=\Sigma \delta \mathrm{d}_{\mathrm{i}}=0.10 \mu \mathrm{m}+2.5 \mathrm{ppm} \cdot \mathrm{d} .
$$

At a distance of $100 \mathrm{~mm}$ corresponding to the extension of the object being measured, the sum of the distance-specific errors is:

$$
8 \mathrm{~d}_{100}=0.35 \mu \mathrm{m}=3.5 \mathrm{ppm} @ 100 \mathrm{~mm} .
$$

\section{Total Distance Error $\Delta d(8 d, \Delta p)$}

Combining the sum of the distance-specific errors, $8 \mathrm{~d}$, with the total position error, $\Delta \mathrm{p}$, over the one-meter travel of the measuring machine stage yields:

$$
\Delta \mathrm{d}=8 \mathrm{~d}+\Delta \mathrm{p}=0.882 \mu \mathrm{m}+2.77 \mathrm{ppm} \times \mathrm{d} \text {. }
$$

For a distance corresponding to the length of the $100-\mathrm{mm}$-long object, the total error in the distance measurement is:

$$
\Delta \mathrm{d}=1.109 \mu \mathrm{m}=1.1 \mathrm{ppm} @ 100 \mathrm{~mm} .
$$




\section{Error in Extension Measurement $\Delta \mathrm{e}$}

In this example, the errors in the coordinate-measuring-machine measurement of extension derive from extension-specific errors associated with boundary location and definition, plus the error in distance measurement.

\section{Boundary-Location Errors}

Boundary-location error in extension measurements represents the systematic failure of the probe to signal the proper location of the material boundary of the object and is of the form:

$$
\delta_{e}=\mp 2 \cdot 8 P_{b v}
$$

where the minus sign is for outside-caliper measurements, the plus sign is for inside-caliper measurements, and $\delta \mathrm{P}_{\mathrm{bz}}$ is positive for location of the arbitrary boundary behind the material boundary.

a. Location of Arbitrary Boundary: Various phenomena give rise to the direction-dependent boundary-location errors inherent to extension measurements which individually contribute to the aggregate effect and $\delta \mathrm{P}_{\mathrm{br}}$ In this example, there is the probe radius (that is, the finite extension of the probe tip along the axis of measurement) and the probe pre-travel (that is, the finite displacement of the probe along the axis of measurement after mechanical contact with the object is made, but before the probe transducer crosses a threshold and signals). Assuming that the gross effects of probe radius and pretravel are eliminated by pre-characterization of the probe, there remain the extension-specific sources of error due, for example, to deformation of the object by mechanical contact and variation of pretravel of the probe with the direction of approach to the object.

Given a three-dimensional touch-trigger probe with a specified pre-travel variation over $360^{\circ}$ in the X-Y plane of $\pm 0.5 \mu \mathrm{m}$, the extension-specific error is taken to be:

$$
8 \mathrm{e}_{b z}=2 \cdot 0.5 \mu \mathrm{m}=1.0 \mu \mathrm{m} .
$$

b. Compensated Boundary Location: The contribution to extension-specific error due to location of an arbitrary boundary can in some situations be compensated by an empirical procedure while in the majority of cases a theoretical model of the probe-object interaction is required. In the example being followed here, the probe is assumed to have a residual directiondependent variation in pretravel of: 


$$
\delta \mathrm{e}_{\mathrm{bt}}=0.1 \mu \mathrm{m} .
$$

\section{Average-Material-Boundary Location}

Extension-specific error due to average-boundary location is associated with local variation in the position of the material boundary of the object such that it has no single extension.

In the example being followed here, the steel block is assumed to have a surface-finish peak-to-valley of $0.1 \mu \mathrm{m}(0.067 \mu \mathrm{m} \mathrm{RMS})$ which when mechanically probed leads to a contribution to extension-specific error:

$$
8 \mathrm{e}_{\mathrm{bt}}=0.2 \mu \mathrm{m} .
$$

\section{Sum of Extension-Specific Errors 8e}

In this example, the total extension-specific error $\delta \mathrm{D}$ is the sum of the errors associated with mislocation of a detected boundary relative to a material boundary and mislocation of a material boundary relative to some simple geometrical shape used to describe the object.

$$
\begin{aligned}
\delta \mathrm{e} & =\Sigma \delta \mathrm{e}_{\mathrm{i}}=\delta \mathrm{e}_{\mathrm{bt}}+\delta \mathrm{e}_{\mathrm{bm}} \\
& =0.2 \mu \mathrm{m}+0.1 \mu \mathrm{m}=0.3 \mu \mathrm{m}
\end{aligned}
$$

Note that extension-specific error is a purely additive error, independent of the magnitude of the extension.

\section{Total Extension Error $\Delta e(8 e, \Delta d)$}

Combining the sum of the extension-specific errors $8 \mathrm{e}$ with the total distance error $\Delta \mathrm{d}$ yields the total extension error:

$$
\Delta \mathrm{e}=8 \mathrm{e}+\Delta \mathrm{d}=1.182 \mu \mathrm{m}+2.77 \mathrm{ppm} \cdot \mathrm{d}
$$

For the $100 \mathrm{~mm}$-long object, the total extension error, computed and rounded to a significant figure, is:

$$
\begin{aligned}
\Delta \mathrm{e}_{100} & =1.459 \mu \mathrm{m} \\
& \rightarrow 1.5 \mu \mathrm{m}=15 \mathrm{ppm} @ 100 \mathrm{~mm} .
\end{aligned}
$$




\section{E. Summary and Analysis of Errors}

This error-budget analysis -- carried out for the case of a commercial-quality displacement-interferometer-equipped coordinate measuring machine used to measure the length of a polished, parallel-faced, $100 \mathrm{~mm}$-long, steel block -illustrates issues specific to the example as well as ones associated with precision dimensional measurements in general. The specific numerical results may be summarized and then analyzed in terms of their relative typehierarchical, additive-multiplicative, and axiom-specific behaviors.

\section{Summary of Numerical Results of Analysis}

Table 7 below summarizes results specific to the hypothetical example being examined. Tabulated are errors specific to the type and total errors for each type, on both an incremental $( \pm \delta \ell)$ and a fractional $( \pm \delta \ell / \ell)$ basis. For comparison, also shown (in italics) is the error in the vacuum wavelength of the laser which provides the reference to the SI unit of length.

Table 7. Summary of Total Errors by Dimensional Type in the Example of the Laser-Interferometer-Based CMM Measurement of Extension of a $100 \mathrm{~mm}$ Block

\begin{tabular}{|c|c|c|c|c|}
\hline \multirow{2}{*}{$\begin{array}{l}\text { Dimensional } \\
\text { Type }\end{array}$} & \multicolumn{2}{|c|}{ Type-Specific Error } & \multicolumn{2}{|c|}{ Total-Error in Type } \\
\hline & Add $8 \ell$ & Mult $8 \mathrm{e} / \mathrm{e}$ & Add $\Delta \ell$ & Mult $\Delta / / \ell$ \\
\hline Extension & $0.3 \mu \mathrm{m}$ & $\cdots$ & $1.462 \mu \mathrm{m}$ & $14.62 \mathrm{ppm}$ \\
\hline Distance & $0.35 \mu \mathrm{m}$ & $3.5 \mathrm{ppm}$ & $1.102 \mu \mathrm{m}$ & $11.02 \mathrm{ppm}$ \\
\hline Position & $0.75 \mu \mathrm{m}$ & $7.5 \mathrm{ppm}$ & $0.812 \mu \mathrm{m}$ & $8.12 \mathrm{ppm}$ \\
\hline Displacement & $0.06 \mu \mathrm{m}$ & $0.6 \mathrm{ppm}$ & $0.062 \mu \mathrm{m}$ & $0.62 \mathrm{ppm}$ \\
\hline Wavelength & $-\ldots$ & $\ldots$ & $0.002 \mu \mathrm{m}$ & $0.02 \mathrm{ppm}$ \\
\hline
\end{tabular}

\section{Analysis of Results}

The particular results of the $\mathrm{CMM} /$ extension-measurement example illustrate a number of more general patterns in dimensional measurements including: a hierarchy of accuracies, dominance by additive errors and multiplicative errors at the short end and long end of the range respectively, and the minimal contribution of laser-wavelength error to total error. 
a. Hierarchy of Accuracies Among the Types: The last column, which gives the total error $\Delta / / \ell$ by type, illustrates the inherent loss of accuracy as one moves through the progression of types of dimensional measurements. Note that the accuracy of the displacement measurements is down by an order of magnitude from that of the reference wavelength. The accuracy of position is down by another order from there, while the accuracy of distance and extension down another factor of two or three further from there. These trends in these relationships are general. On the extremes, displacement measurements always have the best and more readily obtained accuracy and position and distance are intermediate, while extension measurements always have the lowest accuracy and the most dearly bought.

b. The Additive-Multiplicative Errors: The second and third columns, which give the type-specific errors, $\delta \ell$ and $\delta / / \ell$ respectively, illustrate the additive-vsmultiplicative character of errors in the different types. On the extremes, the extension-specific errors are purely additive, which means they dominate at short lengths. In contrast, the displacement-specific errors are almost purely multiplicative, which means they dominate at long ranges.

For the hypothetical CMM of the example, these trends are further illustrated by Table 8 which shows errors in ranges of 10 and $1000 \mathrm{~mm}$. Note especially in the table the near-pure additive $1.2 \mu \mathrm{m}$ (121 ppm) error in extension at $10 \mathrm{~mm}$ compared to the near-pure multiplicative $0.26 \mathrm{ppm}(0.26$ $\mu \mathrm{m}$ ) error in displacement at $1000 \mathrm{~mm}$.

Table 8. Total Error by Dimensional Type at Ranges of 10, 100 and 1000 $\mathrm{mm}$ in the Example of the Laser-Interferometer-Based CMM Measurement of Extension of a Block

Dimension Error@10 mm Error @ 100 mm Error@1000 mm

$\begin{array}{llllll}\text { Extension } & 1.2 \mu \mathrm{m} / 121 \mathrm{ppm} & 1.5 \mu \mathrm{m} / 15 \mathrm{ppm} & 3.9 \mu \mathrm{m} / 4 \mathrm{ppm} \\ \text { Distance } & 0.9 \mu \mathrm{m} / 91 \mathrm{ppm} & 1.2 \mu \mathrm{m} / 11 \mathrm{ppm} & 3.6 \mu \mathrm{m} / 3.6 \mathrm{ppm} \\ \text { Position } & 0.08 \mu \mathrm{m} / 8 \mathrm{ppm} & 0.11 \mu \mathrm{m} / 1 \mathrm{ppm} & 0.35 \mu \mathrm{m} / 0.35 \mathrm{ppm} \\ \text { Displcmnt } & 0.04 \mu \mathrm{m} / 4 \mathrm{ppm} & 0.06 \mu \mathrm{m} / 0.6 \mathrm{ppm} & 0.26 \mu \mathrm{m} / 0.26 \mathrm{ppm}\end{array}$




\section{Axiom-Type Matrix Analysis of Results for the CMM Example}

The error-budget for the CMM measurement of the $100 \mathrm{~mm}$ block serves to illustrate the use of the axiom-type matrix of errors for the analysis of the performance of the overall system of machine, object, and techniques for the achievement of precision dimensions. Table 9 shows in the type-vs-axiom matrix format of Table 5 the results of the CMM-extension error budget summarized in Table 6. Also shown, in italics, are examples of the matrix elements not illustrated by the CMM-extension example.

Table 9. The Axiom-Type Error Matrix for the Example of the LaserInterferometer-Based CMM Measurement of Extension

\begin{tabular}{llll}
\hline \hline Dimension & Error of Zero & Error of Unit & Error of Scale \\
Displacement & $\begin{array}{l}\text { Dead-Path } \\
\text { Thermal-Drift } \\
\text { Least-Count }\end{array}$ & $\begin{array}{l}\text { Laser Wavelength } \\
\text { Mirror-Path Index } \\
\text { Cosine I-vs-L }\end{array}$ & $\begin{array}{l}\text { Polarization Mixing } \\
\text { Grad in MP Index } \\
\text { Bending of MP Ways }\end{array}$ \\
Position & $\begin{array}{l}\text { Probe-Tilt Abbe } \\
\text { Probe-Setting }\end{array}$ & Cosine T-vs-I & Grad in Table Temp \\
Distance & $\begin{array}{l}\text { Point-Definition } \\
\text { Extension }\end{array}$ & $\begin{array}{l}\text { Cosine O-vs-T } \\
\text { Object T-Expansion }\end{array}$ & Bending of Object \\
& $\begin{array}{l}\text { Matl-Av-Bndry } \\
\text { Backlash }\end{array}$ & $\begin{array}{l}\text { Thickness-Dependent Approach to Probe } \\
\text { Probe "Penetration" }\end{array}$ & Resolution Limit \\
& & &
\end{tabular}

a. Errors in the Zero of Each Type: As indicated in Table 9, each of the types of dimensional measurements in the CMM-block example has associated with it a type-specific error of the zero $\left(8 \ell_{2}\right)$ corresponding to a shift of the effective origin of that type. In the displacement measurement, there are the uncompensated change in the index of refraction of the dead-path, the movement of the effective optical-path location of the interferometer reference due to thermal drift, and the uncertainty in the location of the zero due to the least-count of the interferometer system. In the position measurement, there are the non-cumulative change in the location of the position origin due to the tilt of the probe relative to the displacement axis (Abbe error) and the uncertainty in its location due to variations in probe-setting. In the distance measurement, there is the uncertainty in objects geometry which limits 
reduction to centroids, that is, points. Finally, in the extension measurements, there are the arbitrariness of the boundary located relative to the material boundary and the variation of the location of the material boundary about its mean. While not in the example, backlash corresponds to an error of the zero of extension because it only appears with the bi-directional approach to the object.

b. Errors in the Unit of Each Type: Similarly, as indicated in Table 9, each of the types has associated with it a type-specific error of the unit $\left(\delta \ell_{u}\right)$, that is, error proportional to the first-order of the dimension measured. In displacement, there are the error in the value of the vacuum wavelength of the laser, in the index of refraction of the path of the moveable mirror, and the angle of misalignment between the axis of the interferometer (I) and the direction of propagation of the incident light (L). In position measurement, there is the angle of misalignment between the axis of translation of the mirror (T) and the axis of the interferometer (I). In distance measurement, there is angle of misalignment between the object $(\mathrm{O})$ and the probe-path, which for a rigid-body system is the same as the axis of translation of the mirror ( $T$ ). While not discussed in the example, an error in the unit of extension can also arise due to any dependence of the effective "penetration' of the probe (positive or negative) on the thickness of the object.

c. Errors in the Scale of Each Type: Finally, as indicated in Table 9, for each dimensional type, there are type-specific nonlinearities corresponding to errors of the scale $\left(8 q_{41}\right)$. In the displacement measurement, there is the intermixing of the reference- and measuring-beams due to their imperfect separation by polarization states which leads to a non-linearity in the interpolation between fringes. While not in the example, other conditions lead to errors of the scale. In displacement measurements, these include a gradient in the index of refraction along the interferomter mirror path (MP) as well as any lateral bending of the mechanical ways which support the mirror along that path; in position measurements, a gradient in the temperature of the part of the machine which supports the object to be measured; in distance measurements a bending of the object; and in extension measurements, an approach to the resolution limit of the probe.

In sum, this analysis of the hypothetical case of the use of a laser-interferometer-based coordinate measuring machine for measurement of the length of a part has shown the nature and types of the errors that can arise in that specific example. What the example illustrates is that the use of the axiom-type matrix of errors provides as a complete, self-consistent scheme for systematically 
partitioning and analyzing such errors. The approach is, however, more generally useful.

\section{Application of Type-Axiom Error Matrix to Manufacturing Operations}

The axiom-type matrix just applied to the characterization of the dimensionmeasurement process applies equally to the characterization of the dimensiongeneration process, that is, the manufacture-to-design of a dimensionallyspecified product.

a. "Machine Tool" versus "Measuring Machine": Machine tools for the generation of dimensioned forms and measuring machines for the characterization of dimensioned forms share the elements of frame, carriage, scale and "probe," where the formers's probe is for changing the location of material and the latter's "probe" is for ascertaining where the material is located. This commonality is shared by all such "machine tools" - whether metal-cutting lathes, ion-beam milling machines, or photolithographic step-and-repeat cameras - and all such measuring machines - whether they be coordinate measuring machines, measuring microscopes, or micrometer calipers. As such machine tools and measuring machines must conform to the same axiom-type system to carry out their essential functions.

b. Example of Extension Errors in Milling: All the errors indicated in Table 9 for the CMM measurement of a block are also manifest in machine-tool-based manufacture of dimensionally specified products.

Consider briefly, for example, the use of a laser-interferometer-based milling machine for the manufacture of a multiple-finned turbine-blade fan where the part design calls for the machining of equally spaced fins of identical tapering cross-section. In general the machine will be subject to all the sources of error identified in Table 9. Even under the conditions that the machine can generate a cutter path that conforms ideally to the part design (that is, can generate displacement, position and distance perfectly), errors of extension can still occur. Under certain conditions of tool-force and material-stiffness, deflection of the fin by the cutter will vary in proportion to the thickness of the fin at the point of cutting, resulting in error in the material form even for an errorless cutter-path. Thus, while the spacing between fins is perfect (corresponding to no errors of the unit, the zero and the scale in distance), the error in the crosssection of each fin would have a "cooling-tower" shape corresponding to errors in each of the zero, unit and scale of extension and fully analogous to the arbitrary boundary, thickness-dependent probe-penetration and limit-ofresolution errors indicated in Table 9. 


\section{CONCLUSION}

This chapter on precision dimensional measurements for modern manufacturing has presented a new scheme for the assessment of dimensional error, both in measurement processes and in manufacturing processes. The scheme is based on a matrix of dimensional types and measurement axioms which forms a complete, self-consistent system for assessing dimensional error. The scheme has been illustrated by a case-study of a coordinate measuring measurement of an extended object, with the type-axiom related errors indicated as being similarly manifest in manufacturing processes such as the milling of turbine blades. This type-axiom matrix should provide a useful means for the exposure of error, the diagnosis of its cause, and the means of practical elimination through more effective design of machines, processes and techniques for the manufacture of products with precisely dimensioned forms.

\section{ACKNOWLEDGMENTS}

The author wishes to acknowledge the generous contribution and evaluation of ideas contained in this chapter by Drs. Estler, Gilsinn, Phillips, Simpson, Stone, Teague and Vorburger of the Manufacturing Engineering Laboratory of NIST. 


\section{Appendix A. Errors in Dimensional Measurements Due to Angular Misalignments of System Elements}

Accuracy of measurement of dimensions of physical objects by means of a coordinate measurement system based on a laser displacement interferometer, such as the prototypical interferometer shown schematically in a plan-view

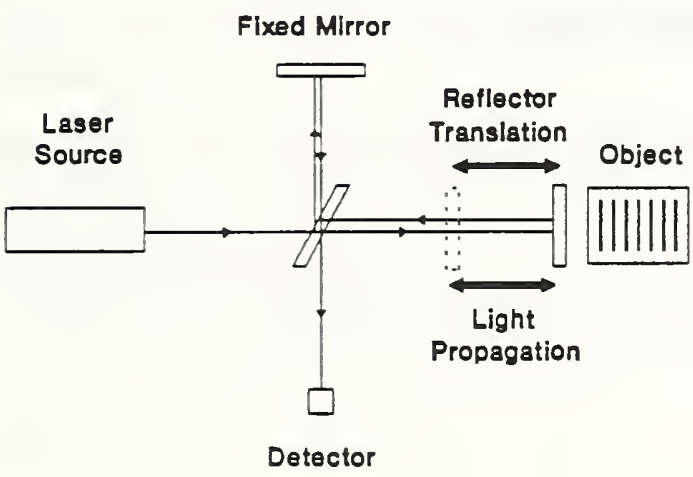
representation here, depends upon precise alignment of the axes of the laser, interferometer, translating mirror, and the object. Errors in measurements of displacement, position, and distance (and/or extension) arise respectively from misalignment of pairs of axes - interferometer and laser, translating mirror and interferometer, and object and translating mirror, as described individually and in combination below.

\section{Misalignment Error Specific to Displacement}

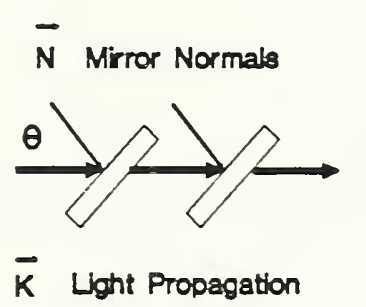

For light of wavelength $\lambda_{0}$ propagating in medium of index of refraction $\mathrm{n}$ in a direction $\mathbf{K}$ at angle $\theta$ to the axis of the interferometer (defined by the normals $\mathbf{N}$ to the reference and moveable reflectors), the change in spacing of the interferometer mirrors, $\Delta \mathrm{s}$, is related to the observed change in fringe order number, $\Delta \mathrm{m}$, by the interferometry equation:

$D=\Delta s=\Delta \mathrm{m} \cdot \lambda_{\alpha} / 2 \mathrm{n} \cdot \cos \theta$.

Note that misalignment between the axis of the interferometer and the axis of propagation of the incident light constitutes an error of the metric or unit of displacement relative to that of the light.

\section{Misalignment Error Specific to Coordinate Position}

For a moveable or "measuring" reflecting surface (plane mirror or retroreflector) which translates at an angle $\phi$ relative to the axis $\mathbf{N}$ of the parallel-plate interferometer, the translation $\mathrm{T}$ is related to the change in spacing $\Delta \mathrm{s}$ of the interferometer by the "cosine-error" equation: 
$\vec{T}$ Mirror Translation

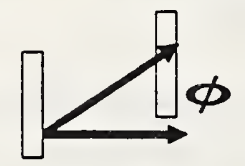

N Mirror Normals

$$
\mathrm{P} \cdot \cos \phi=\mathrm{T} \cdot \cos \phi=\Delta \mathrm{s} .
$$

Note that misalignment of the axis of translation of the measuring element of the interferometer relative the axis of the interferometer constitutes an error of the metric or unit of position (coordinate) relative to that of the displacement.

\section{Misalignment Error Specific to Object-Distance}

$\overrightarrow{\mathrm{T}}$ Translation Axis

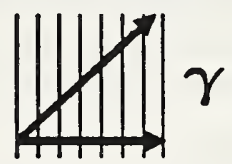

$\overrightarrow{0}$ Object Axis

Finally, given an object with features with an axis $\mathbf{O}$ oriented at an angle $\gamma$ relative to the direction of translation of the measuring mirror of the interferometer, the relation of the distance between features on the object and the translation of the mirror is given by the equation:

$$
\mathrm{d}=\mathrm{T} \cdot \cos \gamma \text {. }
$$

Note that the misalignment of the axis of the object and that of the translation of the measuring mirror constitutes an error in the metric or unit of distance relative to that of position.

\section{Combined Misalignment Error in Distance}

For a system in which there are all three misorientations described above, the relation of the distance between features on the object and the observed change in the fringe order number is given by:

$$
\mathrm{d}=\left(\Delta \mathrm{m} \cdot \lambda_{0} \cdot \cos \gamma\right) /(2 \cdot \mathrm{n} \cdot \cos \theta \cdot \cos \phi) .
$$

\section{Explicit Form for Misalignment Error}

Given the sign convention that the observed distance $d_{o b s}$ is the algebraic sum of the true distance $d_{\text {true }}$ plus the error term $\Delta d$, then:

$$
\begin{aligned}
\Delta \mathrm{d} & =\mathrm{d}_{\text {obs }}-\mathrm{d}_{\text {true }} \\
& =\mathrm{d}-\mathrm{d}(\theta, \phi, \gamma) \\
& =\left(\Delta \mathrm{m} \cdot \lambda_{\mathrm{o}} / 2 \mathrm{n}\right) \cdot[1-\{\cos \gamma /(\cos \theta \cdot \cos \phi)\}]
\end{aligned}
$$


Under the conditions that the angles $\theta, \phi$, and $\gamma$ are each small, successive substitution of the approximations $\cos x \approx 1-\mathrm{x}^{2} / 2$ and $1 /\left(1-\mathrm{x}^{2}\right) \approx 1+\mathrm{x}^{2}$ into Eq. A5 leads to:

$$
\begin{aligned}
\Delta \mathrm{d} & \approx\left(\Delta \mathrm{m} \cdot \lambda_{\mathrm{o}} / 2 \mathrm{n}\right) \cdot\left[1-\left\{\left(1-\gamma^{2} / 2\right) /\left(1-\theta^{2} / 2\right) \cdot\left(1-\phi^{2} / 2\right)\right\}\right] \\
& \approx\left(\Delta \mathrm{m} \cdot \lambda_{\mathrm{d}} / 2 \mathrm{n}\right) \cdot\left[1-\left\{\left(1-\gamma^{2} / 2\right) \cdot\left(1+\theta^{2} / 2\right) \cdot\left(1+\phi^{2} / 2\right)\right\}\right],
\end{aligned}
$$

which with multiplication and retention of terms of the lowest order yields the final result:

$$
\Delta \mathrm{d} \approx\left(\Delta \mathrm{m} \cdot \lambda_{\mathrm{o}} / 2 \mathrm{n}\right) \cdot\left(+\theta^{2}+\phi^{2}-\gamma^{2}\right) / 2
$$

Note that since the first and second terms are negative and the third term is positive, the overall error in distance due to angular misalignments can be either positive or negative and the measured distance can be either greater than or less than the actual.

\section{REFERENCES}

1. E.C.Teague, "Nanometrology", Proc. of Engineering Foundations Conference on Scanning Probe Microscopes: STM and Beyond", Santa Barbara, Jan. 1991, published by The American Physical Society.

2. "Documents Concerning the New Definition of the Metre," Metrologia 19, C. Springer-Verlag, 163-177 (1984).

3. R.C.Quenelle and LJ.Wuerz, "A New Microcomputer-Controlled Laser Dimensional Measurement and Analysis System," Hewlett-Packard Journal, 2-22 (1983).

4. J.S.Beers and K.B.Lee, "Interferometric Measurement of Length Scales at the National Bureau of Standards," Precision Engineering Journal, Vol. 4, No. 4, 205-214 (1982).

5. T.D.Doiron, "High Precision Gaging With Computer Vision Systems," Elsevier, Industrial Metrology 1, $43-54$ (1990).

6. R.I.Scace, "Foreign Trip Report to Japan," Center for Electronics and Electrical Engineering, NIST, Gaithersburg, MD, Report No. 720-46 (1989).

7. E.C.Teague, "The NIST Molecular Measuring Machine Project: Metrology and Precision Engineering Design," Journal of Vacuum Science and Technology B7, 1890-1902 (1989). 
8. C.F.Vezzetti, R.N.Varner, and J.E.Potzick, "Bright-Chromium Linewidth Standard, SRM 476, for Calibration of Optical Microscope Linewidth Measuring Systems," NIST Special Publication, 260-114 (1991).

9. H.P.Layer and W.T.Estler, "Traceability of Laser Interferometric Length Measurements," NBS Technical Note 1248 (1988).

10. C.Eisenhart, "Realistic Evaluation of the Precision and Accuracy of Instrument Calibration Systems", Journal of Research of the National Institute of Standards and Technology, Vol.67C, No.2, April-June 1963.

11. R.Carnap, "Philosophical Foundations of Physics," An Introduction to the Philosophy of Science, ed., Martin Gadner, Basic Books, Inc., New York, London (1966).

12. J.A.Simpson, "Foundations of Metrology," Journal of Research of National Institute of Standards and Technology, Vol. 86, No. 3 (1981).

13. C.R.Steinmetz, "Sub-Micron Displacement Measurement Repeatability on Precision Machine Tools With Laser Interferometry," SPIE Conference on Optical Mechanical and Electro-Optical Design of Industrial Systems, Vol. 959, 178-0192 (1988).

14. F.E.Jones, "The Refractivity of Air," Journal of Research of the National Institute of Standards and Technology, Vol. 86, No. 1 (1981).

15. W.T.Estler, "Laser Interferometry in Length Measurement," NIST Special Publication, (1990).

16. W.T.Estler, "High-accuracy Displacement Interferometry in Air," Applied Optics, Vol. 24, No. 6, 808-815 (1985).

17. C.R.Steinmetz, "Sub-Micron Displacement Measurement Repeatability on Precision Machine Tools With Laser Interferometry," SPIE Conference on Optical Mechanical and Electro-Optical Design of Industrial Systems, Vol. 959, 178-0192 (1988).

18. G.Zhang, R.Veale, T.Charlton, B.Borchardt, and R.Hocken, "Error Compensation of Coordinate Measuring Machines," Annals of the CIRP, Vol. 34, 445-448 (1985).

19. N.A.Duffie and S.J.Malmberg, "Error Diagnosis and Compensation Using Kinematic Models and Position Error Data," Annals of the CIRP, Vol. 36, 355-358 (1987).

20. "Methods for the Performance Evaluation of Coordinate Measuring Machines", American National Standard B89.1.12 (1991), American Society of Mechanical Engineers, NY, NY 10017.

21. M.T.Postek, "Scanning Electron Microscope-based Metrological Electron Microscope System and New Prototype Scanning Electron Microscope Magnification Standard," Scanning Microscopy, Vol. 3, No. 4, 1087-1099 (1989).

22. R. Donaldson, "Error Budgets", in Tech. of Machine Tools, Vol.5, Ser/ 9.14 (1980). 
NIST-114A

(REV. 3-89)
U.S. DEPARTMENT OF COMMERCE NATIONAL INSTITUTE OF STANDARDS AND TECHNOLOGY

\section{BIBLIOGRAPHIC DATA SHEET}

1. PUBLCATION OR REPORT NUMBER NISTIR 4644

2. PERFORMING ORGANIZATION REPORT NUMBER

3. PUBLCATION DATE

AUGUST 1991

\section{TITLE AND SUBTITLE}

NEW CONCEPTS OF PRECISION DIMENSIONAL MEASUREMENT FOR MODERN MANUFACTURING

5. AUTHOR(S)

Dr. Dennis A. Swyt

6. PERFORMING ORGANIZATION (IF JOINT OR OTHER THAN NIST, SEE INSTRUCTIONS) U.S. DEPARTMENT OF COMMERCE NATIONAL INSTITUTE OF STANDARDS AND TECHNOLOGY GAITHERSBURG, MD 20899 7. CONTRACT/GRANT NUMBER

8. TYPE OF REPORT AND PERIOD COVERED

9. SPONSORING ORGANIZATION NAME AND COMPLETE ADDRESS (STREET, CITY, STATE, ZIP)

10. SUPPLEMENTARY NOTES

DOCUMENT DESCRIBES A COMPUTER PROGRAM; SF-185, FIPS SOFTWARE SUMMARY, IS ATTACHED.

11. ABSTRACT (A 200-WORD OR LESS FACTUAL SUMMARY OF MOST SIGMIFICANT INFORMATION. IF DOCUMENT INCLUDES A SIGNIFICANT BIBLOGRAPHY OR UTERATURE SURVEY, MENTION IT HERE.)

A new scheme for the analysis of the origin and propogation of errors in length-based dimensions is presented, one which is applicable to the characterization of measuring machine, machine tools, and the dimensioned parts which they characterize or form. The scheme involves a matrix of four dimensional-type-specific errors (associated with the length-based quantities of displacement, position, distance and extension) and three measurement-axiom-specific errors (associated with rules governing the zero, the unit and the scale of measurement). Application of the scheme is illustrated by an propogation of error analysis of the use of a laser-interferometer-based coordinate measuring machine for the determination of the length of a simple part. Also indicated is how an identical analysis applies to the characterization of the performance of machine tools which shape such parts.

12. KEY WORDS (6 TO 12 ENTRIES; ALPHABETICAL ORDER; CAPITALZE ONLY PROPER MAMES; AND SEPARATE KEY WORDS BY SEMICOLONS) accuracy; Coordinate Measuring Machines; dimensional measurement; error analysis; manufacturing; precision

\section{AVAILABIUTY}

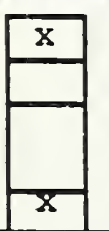

FOR OFFICIAL DISTRIBUTION. DO NOT RELEASE TO NATIONAL TECHMICAL INFORMATION SERVICE (NTIS).

ORDER FROM SUPERINTENDENT OF DOCUMENTS, U.S. GOVERAMENT PRINTING OFFICE, WASHINGTON, DC 20402.

ORDER FROM MATIONAL TECHNICAL INFORMATION SERVICE (NTIS), SPRINOFIEL, VA 22161.

14. NUMBER OF PAINTED PAGES 59

15. PAICE

A04 

